\title{
Star-forming protoclusters associated with methanol masers
}

\author{
V. Minier ${ }^{1,2}$, M. G. Burton ${ }^{2}$, T. Hill ${ }^{2}$, M. R. Pestalozzi ${ }^{3}$, C. R. Purcell ${ }^{2}$, G. Garay ${ }^{4}$, A. J. Walsh ${ }^{5}$, and S. Longmore ${ }^{2}$ \\ 1 Service d'Astrophysique, DAPNIA/DSM/CEA CE de Saclay, 91191 Gif-sur-Yvette, France \\ e-mail: Vincent.Minier@cea.fr \\ 2 School of Physics, University of New South Wales, Sydney 2052, NSW, Australia \\ 3 Onsala Space Observatory, 43992 Onsala, Sweden \\ 4 Departamento de Astronomía, Universidad de Chile, Casilla 36-D, Santiago, Chile \\ 5 Center for Astrophysics, 60 Garden Street, Cambridge, MA, 02138, USA
}

Received 21 April 2004 / Accepted 16 September 2004

\begin{abstract}
We present a multiwavelength study of five methanol maser sites which are not directly associated with a strong (>100 mJy) radio continuum source: G 31.28+0.06, G 59.78+0.06, G 173.49+2.42 (S231, S233IR), G 188.95+0.89 (S252, AFGL5180) and G 192.60-0.05 (S255IR). These radio-quiet methanol maser sites are often interpreted as precursors of ultracompact H II regions or massive protostar sites. In this work, the environment of methanol masers is probed from mid-IR to millimetre wavelengths at angular resolutions of $8^{\prime \prime}-34^{\prime \prime}$. Spectral energy distribution (SED) diagrams for each site are presented, together with mass and luminosity estimates. Each radio-quiet maser site is always associated with a massive ( $\left.>50 M_{\odot}\right)$, deeply embedded $\left(A_{\mathrm{v}}>40 \mathrm{mag}\right)$ and very luminous $\left(>10^{4} L_{\odot}\right)$ molecular clump, with $L_{\text {total }} \propto M_{\mathrm{gas}}^{0.75}$. These physical properties characterise massive star-forming clumps in earlier evolutionary phases than $\mathrm{H}$ II regions. In addition, colder gas clumps seen only at mm-wavelengths are also found near the methanol maser sites. These colder clumps may represent an even earlier phase of massive star formation. These results suggest an evolutionary sequence for massive star formation from a cold clump, seen only at mm wavelengths, evolving to a hot molecular core with a two-component SED with peaks at far-IR and mid-IR wavelengths, to an (ultra-compact) H II region. Alternatively, the cold clumps might be clusters of low-mass YSOs, in formation near the massive star-forming clusters. Finally, the values of the dust grain emissivity index $(\beta)$ range between 1.6 and 1.9.
\end{abstract}

Key words. masers - stars: formation - ISM: dust, extinction

\section{Introduction}

Class II methanol masers were originally detected toward regions of massive star formation (MSF) because many searches at 6.7 and $12.2 \mathrm{GHz}$ had focused on observing regions exhibiting typical MSF signatures, such as strong radio continuum sources (i.e. H II regions), IRAS sources with IR colours of ultra-compact $\mathrm{H}$ II (UC H II) regions ${ }^{1}$ and $\mathrm{OH}$ masers (e.g. Batrla et al. 1987; Schutte et al. 1993; Caswell et al. 1995). At low angular resolution $\left(\sim 1^{\prime}-10^{\prime}\right)$, many 6.7 and $12.2-\mathrm{GHz}$ methanol masers appeared to be associated with $\mathrm{H}$ II regions.

In contrast, high angular resolution $\left(\sim 0.01^{\prime \prime}-1^{\prime \prime}\right)$ observations have demonstrated that methanol masers are in fact generally not co-spatial with strong radio sources (e.g. UC H II regions), but frequently tend to be isolated from them. Out of $\sim 250$ methanol maser sites studied, 75\% are not directly associated (within $0.1 \mathrm{pc}$ ) with UC H II regions or more extended radio sources (Caswell 1996; Walsh et al. 1998; Phillips et al. 1998; Minier et al. 2001). Very weak (<4 mJy) radio continuum emission has, however, been detected toward isolated methanol

1 In this paper, a UC H II region is defined as a region ionised by high-mass stars, with a diameter $\sim 0.1 \mathrm{pc}$ and an electron density $\geq 10^{4} \mathrm{~cm}^{-3}$ producing a $22-\mathrm{GHz}$ flux of $\sim 100 \mathrm{mJy}$ at a distance of $5 \mathrm{kpc}$ (Kurtz et al. 2000). maser sites (e.g. van der Walt et al. 2003). An extreme case is IRAS $20126+4104$ where the methanol maser in the hot molecular core is associated with a $0.1-\mathrm{mJy}$ radio source at $8.6 \mathrm{GHz}$ (Hofner et al. 1999; Minier et al. 2001). This might be the first sign that some isolated methanol masers are associated with precursors of UC HII regions, i.e. protostellar objects on the way to forming a star or a multiple star system. Methanol maser sites are therefore generally radio-quiet in terms of continuum emission $(<1 \mathrm{mJy}$ at $5 \mathrm{kpc}$, i.e. a typical observational sensitivity limit).

Complementary spectral line observations confirm this view. Preliminary results from a molecular line survey with the Mopra millimetre telescope suggest that isolated methanol masers are possibly associated with hot molecular cores (HMCs) (Purcell et al. 2004), which are presumed to be the sites where massive protostars evolve to form an ionising ZAMS star (Kurtz et al. 2000; Fontani et al. 2004). The intense and complex chemistry, the temperature (some $100 \mathrm{~K}$ ) and the density $\left(\geq 10^{7} \mathrm{~cm}^{-3}\right)$ of these HMCs would provide suitable conditions for methanol masers to arise (see Minier et al. 2003, and references therein).

Finally, recent ATCA observations by Minier et al. (2003) might indicate an exclusive association between methanol 
masers and massive star-forming regions following a nil detection rate of masers toward regions of low-mass star formation. All these elements tend to demonstrate that methanol masers may trace an early stage of MSF. Whether methanol masers trace embedded massive protostars or suitable conditions nearby a site of MSF remains an open question.

This paper is devoted to a multiwavelength study of five radio-quiet methanol maser sites and their environments, which are particularly good candidates for hosting a massive protostar (Minier et al. 2000, 2001): G 31.28+0.06, G 59.78+0.06, G 173.49+2.42 (S231， S233IR), G 188.95+0.89 (S252, AFGL 5180) and G 192.60-0.05 (S255IR). Comparisons of images taken from optical to radio wavelengths are presented, accompanied by estimates of the mass, luminosity and temperature of the MSF clumps in each region. We will demonstrate that the five radio-quiet maser sites coincide with massive and luminous star-forming clumps. Finally, it will be shown that methanol masers trace very young clusters of star formation in the earliest stages of their evolution.

Section 2 describes the observations and data archives used for this work. Derived physical quantities and diagrams of spectral energy distributions are presented in Sect. 3, followed by a discussion in Sect. 4 and conclusions in Sect. 5 .

\section{Observations, data archives and analysis}

\subsection{Optical image archives}

An optical image of an $8^{\prime} \times 8^{\prime}$ region was generated around each source using the Digitized Sky Survey (DSS) database (http://archive.eso.org/dss/dss). The Digitized Sky Survey comprises a set of all-sky photographic surveys conducted with the Palomar and UK Schmidt telescopes. The objective was to visualise any association with optical H II regions or main sequence $\mathrm{OB}$ stars after identification with the SIMBAD catalogues on $C D S$.

\subsection{Mid- and far- infrared image archives}

The mid-infrared (mid-IR) and far-infrared (far-IR) images were obtained from the Midcourse Space Experiment (MSX) and from the InfraRed Astronomical Satellite (IRAS) data archives. The values for the mid-IR fluxes were estimated from the MSX images using the KARMA ${ }^{2}$ package, by estimating the flux in the source and also in the background. The background residual flux was measured by taking the averaged flux in boxes at diverse positions around the mm source. This method allows removal of the background noise as well as emission from nearby objects and extended regions. To avoid confusion with silicate emission/absorption at $8.7 \mu \mathrm{m}$ (in the MSX A-band) and with the more extended PAH contribution at $7.7,8.6$ and $11.3 \mu \mathrm{m}$ (in the $A$ - and $C$-bands), the emission contours in the $E$-band $(21.3 \mu \mathrm{m})$ were used to estimate the extent of the thermal dust emission. The source flux then had the background subtracted, and the value was converted

\footnotetext{
${ }^{2}$ See: http://www.atnf.csiro.au/computing/ software/karma/
}

from $\mathrm{W} \mathrm{m}^{-2} \mathrm{sr}^{-1}$ to $\mathrm{Jy}$. The image sensitivity varies from 1.9 to $29.8 \times 10^{-7} \mathrm{~W} \mathrm{~m}^{-2} \mathrm{sr}^{-1}$, with an angular resolution of $18^{\prime \prime}$ (i.e. $0.18 \mathrm{pc}$ at $2 \mathrm{kpc}$ ). The positions in the MSX point source catalogue are accurate to within $1^{\prime \prime}-5^{\prime \prime}$. The conversion factors were $6.84 \times 10^{3}, 2.74 \times 10^{4}, 3.08 \times 10^{4}$ and $2.37 \times 10^{4} \mathrm{Jy}$ per $\mathrm{W} \mathrm{m}^{-2} \mathrm{sr}^{-1}$ for the $A$-band $(8.3 \mu \mathrm{m}), C$-band $(12.1 \mu \mathrm{m})$, $D$-band $(14.6 \mu \mathrm{m})$ and $E$-band $(21.3 \mu \mathrm{m})$, respectively (see Egan et al. 1999 for a MSX explanatory guide). These factors include a correction turning the 6-arcsec square pixel into a Gaussian area. The far-IR fluxes were taken directly from the IRAS Point Source Catalogue (IRAS PSC).

\subsection{Submillimetre and millimetre continuum observations}

The submillimetre observations at 450 and $850 \mu \mathrm{m}$ were conducted using the Sub-mm Common User Bolometer Array (SCUBA) on the James Clerk Maxwell Telescope (JCMT) in September 2002 for three of the five sources. G 31.28+0.06 was observed by Walsh et al. (2003). The observations were made in jiggle map mode with a map size $\sim 2-3$ arcmin. The pixel size was 3 arcsec. Pointing and calibration were made on HL Tau. The average zenith atmospheric optical depths were 1.8 and 0.3 at 450 and $850 \mu \mathrm{m}$, respectively. The volt-to-Jy-beam ${ }^{-1}$ conversion factors were 373.1 and 260.0. The image sensitivities were $\sim 0.3$ and $0.01 \mathrm{Jy} \mathrm{beam}^{-1}$ at 450 and $850 \mu \mathrm{m}$, respectively. The $F W H M$ beam sizes were estimated to be 8 and 15 arcsec (i.e. $0.08-0.15 \mathrm{pc}$ at $2 \mathrm{kpc}$ ) at 450 and $850 \mu \mathrm{m}$, respectively. The data were reduced with the standard procedure SURF (reduceswitch, flatfield, extinction, remsky and rebin). The flux density values were estimated in each image with the KVIEW procedure described in Sect. 2.2.

The 1.2-mm observations were made using the SEST IMaging Bolometer Array (SIMBA) on the Swedish-ESO Submillimetre Telescope (SEST) in October 2001 and June 2002. SIMBA is a 37-channel hexagonal array in which the $H P B W$ of a single element is about $24^{\prime \prime}$ (i.e. $0.24 \mathrm{pc}$ at $2 \mathrm{kpc}$ ) and the separation between elements on the sky is $44^{\prime \prime}$. The bandwidth in each channel is about $50 \mathrm{GHz}$. Spectral line emission present in the band may contribute to the total continuum flux values reported in this paper, though not more than $30 \%$. Free-free emission is not expected to contribute to the (sub-)mm flux given the radio-quiet nature of the targets. Assuming optically thin free-free emission for frequencies $\geq 8 \mathrm{GHz}, 80 \mathrm{mJy}$ at $8.6 \mathrm{GHz}$ would convert to $57 \mathrm{mJy}$ at $250 \mathrm{GHz}$, i.e. $\sim 1 \%$ of the $1.2-\mathrm{mm}$ flux measured at the same position. Similarly, for an ionized wind model with $v^{0.6}$ (Panagia \& Felli 1975), the extrapolation at $250 \mathrm{GHz}$ of the radio fluxes would yield less than $11 \%$ of the observed flux densities. These observations were made in fast-mapping mode. Typical areas of $600^{\prime \prime} \times 384^{\prime \prime}$ were imaged in order to detect nearby IRAS sources in the field, using a scan speed of $80^{\prime \prime} \mathrm{s}^{-1}$. The resulting pixel size is 8 arcsec. Typically, total observation times were around 15 minutes per source, giving a sensitivity $\sim 0.1 \mathrm{Jy} \mathrm{beam}^{-1}$. Zenith opacities ranged from $0.2-0.4$ throughout the observing runs in 2001 and 2002. The resulting data were reduced with the MOPSI mapping software package 
developed by R. Zylka, using the "deconvolution" algorithm ${ }^{3}$ to remove the contribution of the electronics arising from the fast-mapping observing mode, the "converting" algorithm (Salter 1983) to convert the coordinates from rectangular to equatorial, and partly the NOD2 and GILDAS libraries. The calibration of our data was performed using Uranus. The multiplication factor between counts and $\mathrm{Jy}_{\text {beam }}^{-1}$ was $138.8 \mathrm{mJy} \mathrm{count}^{-1}$ beam $^{-1}$ for the October 2001 run (G 188.95+0.89, G 192.60-0.05) and $66.9 \mathrm{mJy}_{\text {count }}{ }^{-1}$ beam $^{-1}$ for the June 2002 run $(\mathrm{G} 31.28+0.06, \mathrm{G} 59.78+0.06)$. The flux density values were estimated with the KVIEW procedure described in Sect. 2.2. The 1.2-mm fluxes for G $173.49+2.42$ were taken from Beuther et al. (2002).

\subsection{Molecular spectral line observations}

The molecular line observations were carried out in May 1999 with the Onsala-20 m millimetre telescope (OSO-20 m) and in October 2002 with the Mopra millimetre telescope. $\mathrm{CH}_{3} \mathrm{CN}$ $(92 \mathrm{GHz})$ and $\mathrm{C}^{18} \mathrm{O}(109 \mathrm{GHz})$ lines were observed toward each methanol maser position with a single pointing. $80 \mathrm{MHz}$ and $64 \mathrm{MHz}$ bandwidths were used at Onsala and Mopra, respectively. The gain of the Onsala- $20 \mathrm{~m}$ telescope is $\sim 20 \mathrm{Jy} \mathrm{K}^{-1}$ in the range $85-112 \mathrm{GHz}$ while the gain of the Mopra telescope is about $30 \mathrm{Jy} \mathrm{K}^{-1}$. $\mathrm{CH}_{3} \mathrm{CN}$ observations were performed with Mopra only. $\mathrm{C}^{18} \mathrm{O}$ observations were carried out with Mopra toward G 192.60-0.05 and with the OSO-20 m toward the other four sources. The pointing errors were estimated to be less than 5 arcsec for the OSO-20 $\mathrm{m}$ and less than 10 arcsec for Mopra. The data were processed with XS, the Onsala data reduction software.

\subsection{Radio continuum data archives}

Radio continuum information was obtained from Aladin and $S I M B A D$ on $C D S$ within a $4^{\prime} \times 4^{\prime}$ region around each methanol maser site. Specific radio survey catalogues were also searched using VizieR. These included the 1.4-GHz NRAO VLA sky survey (NVSS, Condon et al. 1998), the 5-GHz VLA survey of the Galactic plane (Becker et al. 1994), the survey of radio H II regions in the Northern Sky through recombination line emission (Lockman 1989), the GB6 catalogue of radio sources at $4.85 \mathrm{GHz}$ (Gregory et al. 1996), the UC H II region survey (Kurtz et al. 1994) and an ATCA survey at $8.6 \mathrm{GHz}$ (Walsh et al. 1998). Source positions from Snell \& Bally (1986) for G 192.60-0.05 and from Sridharan et al. (2002) for G 59.78+0.06 were also plotted.

\section{Results}

\subsection{Derivation of physical quantities}

The goal of this paper is to derive the physical characteristics of the methanol maser sites. These physical quantities include the luminosity $\left(L_{\text {total }}\right)$, the gas mass $\left(M_{\mathrm{gas}}\right)$, the gas column density $\left(N_{\mathrm{H}_{2}}\right)$, the gas density $\left(n_{\mathrm{H}_{2}}\right)$, the dust $\left(T_{\text {cold }}\right.$ and $\left.T_{\text {hot }}\right)$ and

\footnotetext{
3 Developed within the SIMBA collaboration.
}

gas temperatures $\left(T_{\text {rot }}\right)$, the virial mass $\left(M_{\text {vir }}\right)$ and the power law index of the dust optical depth $(\beta)$. The values of these physical quantities are derived from the dust continuum emission and from the molecular line emission for each clump in the five MSF regions studied in this paper. We do not intend to model density and temperature structures in detail. This will be undertaken with higher angular resolution instruments in the future.

\subsubsection{Spectral Energy Distribution of dust emission}

The Spectral Energy Distribution (SED) of each millimetre source with continuum data points from $8 \mu \mathrm{m}$ to $1.2 \mathrm{~mm}$ is fitted with two grey-body functions modified with a nonconstant emissivity function, which model emission from a hot $(>100 \mathrm{~K})$ dust core (diameter, $d_{\text {hot }}$ ) embedded in a larger cold $(<30 \mathrm{~K})$ or warm $(30<T<100 \mathrm{~K})$ envelope (diameter, $\left.d_{\text {cold }}\right)$. The dust optical depth in the emissivity function is expressed as a power law $\tau_{\mathrm{d}}=\tau_{0}\left(v / v_{0}\right)^{\beta}$ where $v$ is the frequency and $\beta$ is the dust grain emissivity index. The hot dust core is characterised by a dust temperature $\left(T_{\text {hot }}\right)$ and a solid angle $\left(\Omega_{\text {hot }}\right)$ which are two free parameters in the first grey-body model. The cold (or warm) envelope is characterised by $T_{\text {cold }}$ and $\Omega_{\text {cold }}$ in the second grey-body function. Two additional parameters, the turn-over frequency between optically thin and thick regimes ( $v=v_{0}$ where $\tau=\tau_{0}=1$ ) and $\beta$, are also estimated. In total six free parameters are therefore estimated using a minimum $\chi^{2}$-Levenberg-Marquardt bestfit of the functions to the data.

This model is a good approximation if each methanol maser site harbours a single young stellar object (YSO). In the case of a multiple YSO system or a YSO cluster, our model will reproduce emission from both the individual YSOs and the cluster envelope. However, only a few massive YSOs are expected to be present within each methanol maser site due to the relatively small region observed. The SED will be dominated by emission from the most luminous objects present. In summary, our model will give the SED of a single YSO at best and the total SED of a YSO cluster at least.

The total luminosity $\left(L_{\text {total }}\right)$ is derived by integrating the SED over the frequency/wavelength range. The gas mass is estimated using the following equation, adopting the RayleighJeans approximation to the Planck function, and assuming optically thin emission at $1.2 \mathrm{~mm}$ :

$M_{\text {gas }}=5 \times 10^{-31} \frac{F_{1.2 \mathrm{~mm}} D^{2} \lambda^{2}}{2 k T_{\text {cold }} R_{\mathrm{d}} K_{\mathrm{d}}}$ in $M_{\odot}$,

where $F_{1.2} \mathrm{~mm}$ is the $1.2-\mathrm{mm}$ continuum intergrated flux in $\mathrm{W} \mathrm{m} \mathrm{m}^{-2} \mathrm{~Hz}^{-1}, D$ the distance to the source in $\mathrm{m}, \lambda$ the wavelength in $\mathrm{m}, k$ the Boltzmann's constant in $\mathrm{J} \mathrm{K}^{-1}, T_{\text {cold }}$ the dust temperature in $\mathrm{K}, R_{\mathrm{d}}$ the dust-to-gas mass ratio and $\kappa_{\mathrm{d}}=$ $\kappa_{0}\left(v / v_{0}\right)^{\beta}$ the mass coefficient absorption $\left(\propto \tau_{\mathrm{d}}\right)$ in $\mathrm{m}^{2} \mathrm{~kg}^{-1}$. The Rayleigh-Jeans approximation holds as long as the temperature is greater than $40 \mathrm{~K}$ at $1.2 \mathrm{~mm}$ or $250 \mathrm{GHz}$. For temperatures less than $30 \mathrm{~K}$, the use of the full Planck function is recommended. The values of $\kappa_{\mathrm{d}}$ and $R_{\mathrm{d}}$ may vary with physical conditions. Values of $0.1 \mathrm{~m}^{2} \mathrm{~kg}^{-1}$ at $\lambda=1.2 \mathrm{~mm}$ and $1 \%$ are adopted for $\kappa_{\mathrm{d}}$ and $R_{\mathrm{d}}$, respectively, in the present work (Ossenkopf $\&$ Henning 1994). This gives values of $M_{\text {gas }}$ similar or down 
to 4 times less than when using the mass estimate method from Hildebrand (1983).

$N_{\mathrm{H}_{2}}$ and $n_{\mathrm{H}_{2}}$ are calculated by dividing $M_{\text {gas }}$ by $\pi\left(\theta_{\text {sest }} D / 2\right)^{2}$ and $4 \pi\left(\theta_{\text {sest }} D / 2\right)^{3} / 3$, respectively, where $\theta_{\text {sest }} \approx 24^{\prime \prime}$ is the angular resolution of SEST at $1.2 \mathrm{~mm}$. The values of the column density and density in Table 5 are therefore beam averaged values. $N_{\mathrm{H}_{2}}$ and the $\mathrm{H}_{2}$ mass density are also estimated using $d_{\text {cold }}$, which is the fitted diameter of the protostellar envelope (Tables 5 and 6).

\subsubsection{Molecular line analysis}

The molecular lines in the $\mathrm{CH}_{3} \mathrm{CN}$ and $\mathrm{C}^{18} \mathrm{O}$ spectra are fitted with Gaussian functions. The Gaussian parameters are given in Tables 3 and $4 . \mathrm{CH}_{3} \mathrm{CN}\left(5_{K}-4_{K}\right)$ spectral lines corresponding to different $K$-components are used to derive an estimate of the gas temperature using the rotational diagram method and assuming that $\mathrm{CH}_{3} \mathrm{CN}$ emission is optically thin. The rotation method is based on three assumptions. ( $i)$ The spectral line is optically thin. (ii) The brightness temperature $\left(T_{\mathrm{bg}}\right)$ of the background is negligible with respect to the brightness temperature $\left(T_{\mathrm{b}}\right)$ of the source. (iii) The population of all observed energy levels is described by a single Boltzmann temperature $T_{\text {rot }}$.

Using the equation derived in Nummelin (1998) and the above assumptions, the rotation diagram is a plot of the left hand side quantity in Eq. (2) versus $E_{u} / k$.

$\ln \left(\frac{8 \pi k v_{u l}^{2}}{h c^{3} A_{u l} g_{u}} \int T_{\mathrm{b}} \mathrm{d} v\right)=\ln \left(\frac{N}{Q(T)}\right)-\frac{E_{u}}{k T_{\text {rot }}}$,

where $v_{u l}$ is the frequency of the rotational transition, $h$ is Planck's constant, c is the speed of light, $A_{u l}$ is the absorption coefficient, $g_{u}$ is the degeneracy of the upper energy level, $N$ is the total $\mathrm{CH}_{3} \mathrm{CN}$ column density and $Q(T)$ is the partition function. If the hypotheses were correct, the data points would fall along a straight line (see Fig. 1) whose inverse of the negative slope is the rotational temperature $\left(T_{\text {rot }}\right)$. If all transitions are thermalised, the derived temperature is an estimate of the kinematic temperature of the molecular gas. Note that $T_{\mathrm{b}}$ is calculated from $T_{\mathrm{A}}^{*}$, the antenna temperature as follows:

$T_{\mathrm{b}}=\frac{T_{\mathrm{A}}^{*}}{\eta_{\mathrm{mb}} \eta_{\mathrm{bf}}}$,

where $\eta_{\mathrm{mb}}$ is the main-beam efficiency factor and $\eta_{\mathrm{bf}}$ is the beam-filling factor. In this work, we assume $\eta_{\mathrm{bf}}=1$ (beamaveraged) i.e. $T_{\mathrm{mb}}=T_{\mathrm{b}}$ and $\eta_{\mathrm{mb}}=0.55$ for the OSO-20 $\mathrm{m}$ and $\eta_{\mathrm{mb}}=0.7$ for Mopra.

The $\mathrm{C}^{18} \mathrm{O}$ line is a very useful tool for estimating the $\mathrm{H}_{2}$ column density. In the optically thin regime the $\mathrm{C}^{18} \mathrm{O}$ column density is given by Bourke et al. (1997):

$$
\begin{aligned}
N_{\mathrm{C}^{18} \mathrm{O}}= & 2.42 \times 10^{14} \frac{T_{\mathrm{ex}}+0.88}{1-\exp \left(-5.27 / T_{\mathrm{ex}}\right)} \\
& \times \frac{1}{J\left(T_{\mathrm{ex}}\right)-J\left(T_{\mathrm{bg}}\right)} \int T_{\mathrm{mb}}\left(\mathrm{C}^{18} \mathrm{O}\right) \mathrm{d} v{\text { in } \mathrm{cm}^{-2},}^{-2}
\end{aligned}
$$

where $J(T)=h v / k(\exp (h v / k T)-1), T_{\text {ex }}$ the excitation temperature, $T_{\mathrm{bg}}$ the background temperature and $h$ is Planck's constant.
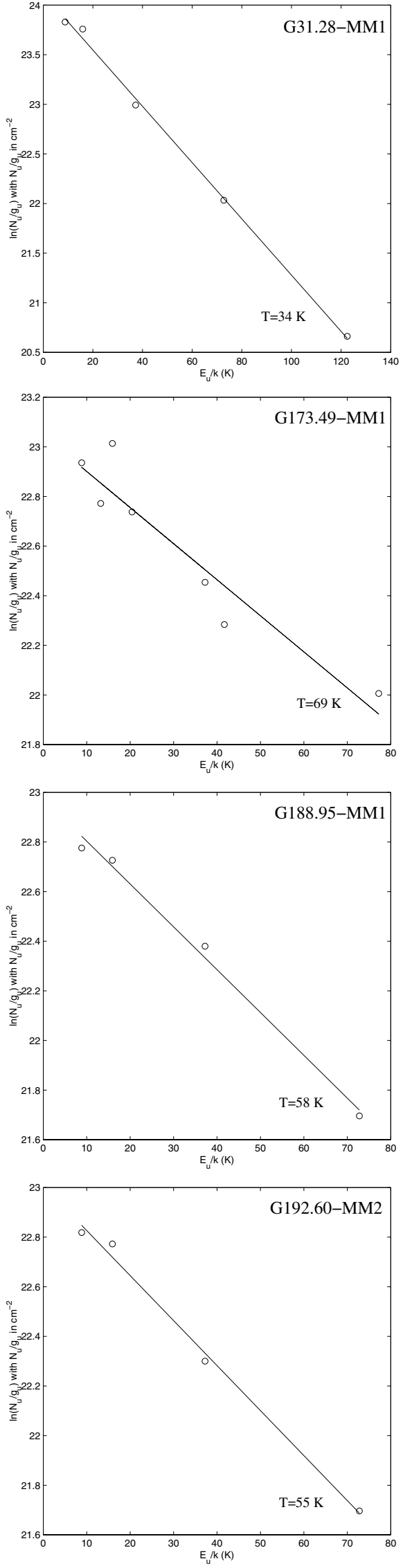

Fig. 1. Rotational diagrams where $\ln \left(N_{u} / g_{u}\right)$ represents the left-hand side of Eq. (2). 
Once $N_{\mathrm{C}^{18} \mathrm{O}}$ is known, $N_{\mathrm{H}_{2}}$ is derived using the column density relationship in Frerking et al. (1982):

$N_{\mathrm{H}_{2}}=5.9 \times 10^{6} N_{\mathrm{C}^{18} \mathrm{O}}+1.3 \times 10^{21} \mathrm{in} \mathrm{cm}^{-2}$.

LTE is assumed and $T_{\mathrm{ex}}=T_{\text {rot }}$. In this way, the rotational temperatures deduced from the $\mathrm{CH}_{3} \mathrm{CN}$ data is used to derive the $\mathrm{C}^{18} \mathrm{O}$ column density. The deduced $\mathrm{H}_{2}$ column densities are representative for the $\mathrm{CH}_{3} \mathrm{CN}$ emitting regions if their angular sizes are similar to that of $\mathrm{C}^{18} \mathrm{O}$ emitting regions. These assumptions will be discussed further in Sect. 4. The total $\mathrm{H}_{2}$ mass is derived by multiplying $N_{\mathrm{H}_{2}}$ by $\pi\left(\theta_{\mathrm{oso}} D / 2\right)^{2}$, where $\theta_{\text {oso }} \approx 34^{\prime \prime}$ is the angular resolution of OSO- $20 \mathrm{~m}$ at $109.8 \mathrm{GHz}$. The density is estimated by dividing the total mass by a source volume $V=4 / 3 \pi\left(\theta_{\mathrm{oso}} D / 2\right)^{3}$. $M_{\mathrm{gas}}$ and $n_{\mathrm{H}_{2}}$ are beam averaged values, i.e. a lower limit of the actual values if the $\mathrm{C}^{18} \mathrm{O}$ cloud has an angular size lower than $\theta_{\text {oso }}$.

\subsubsection{Virial mass}

The virial estimate of the molecular mass follows the approach by MacLaren et al. (1988) with density profile in $R^{-2}$, where $R$ is the radius. The virial mass is given by:

$M_{\text {vir }}=\frac{\sigma^{2} R}{G}=126 \Delta v^{2} R$ in $M_{\odot}$,

where $\sigma$ is the full 3-dimensional velocity dispersion, $R$ is the radius of the cloud in pc, $\Delta v$ is the $F W H M$ of the $\mathrm{C}^{18} \mathrm{O}$ line in $\mathrm{km} \mathrm{s}^{-1}$ and $G$ is the gravitational constant. In this work, $\Delta v=\Delta v_{\mathrm{C}^{18} \mathrm{O}}$ and $R=\theta_{\mathrm{oso}} D / 2$. A molecular clump is gravitationally bound as long as $M_{\mathrm{gas}} / M_{\mathrm{vir}}>1$. Due to uncertainties in the mass estimate, the relation $M_{\text {gas }} / M_{\text {vir }}>1$ will be discussed for each object. Note that the values of $M_{\text {vir }}$ in Table 5 should be divided by $1.4\left(=34^{\prime \prime} / 24^{\prime \prime}\right)$ before comparison with $M_{\text {gas }}$ derived from the dust continuum observations.

\subsection{Presentation of the results}

For each methanol maser site, an overview of the region consisting of a (sub)mm contour map and symbols representing radio sources, IRAS and maser sources overlaid on an optical image is given. This is image a in Figs. 2-6. A close-up image of the methanol maser site is also presented with the midIR and $450 \mu \mathrm{m}$ contours overlaid on the $850 \mu \mathrm{m}$ image. This is image b in Figs. 2-6. For the mm emission sources with enough data points from mid-IR to mm wavelengths (Table 1), the Spectral Energy Distribution (SED) diagrams are presented with the physical parameters and derived physical quantities. IRAS fluxes were used only when an association with a mm source was clearly identified (Table 2). These are plots $\mathbf{c}$ in Figs. 2-6. Finally, $\mathrm{CH}_{3} \mathrm{CN}$ and $\mathrm{C}^{18} \mathrm{O}$ spectra are presented in Figs. 2-6 d with corresponding data values in Tables 3 and 4. Rotational diagrams are given in Fig. 1. Table 5 summarises the derived physical quantities for the five methanol maser sites. To derive these values, the OSO and SEST telescope beams are mainly used as physical angular sizes because $\mathrm{C}^{18} \mathrm{O}$ line emission and dust emission may not arise exactly from the same region. An estimate of the clump diameter in the SED model is given in Figs. 2-6.

\subsection{1. $\mathrm{G} 31.28+0.06$}

Three mm sources are detected toward G 31.28+0.06 (Fig. 2a). They are distributed along a dusty filament seen with SIMBA (Fig. 2a). The methanol maser site coexists with a HII region and an IRAS source in the mm source G31.28+0.06MM1 (hereafter G31.28-MM1), but is separated from the radio source peak emission by $3.5 \times 10^{4} \mathrm{AU}$ at a distance of $5.6 \mathrm{kpc}$. The morphology of the H II region is irregular and clumpy, with a major axis of about $10^{\prime \prime}$. From the values given by Kurtz et al. (1994), the peak brightness temperatures are only $\sim 80 \mathrm{~K}$ at $3.6 \mathrm{~cm}$ and $\sim 55 \mathrm{~K}$ at $2 \mathrm{~cm}$. This implies that this $\mathrm{H}$ II region has a low value EM, and does not belong to the class of UC H II regions as defined by Kurtz et al. (2000). The number of ionizing photons required to excite the $\mathrm{H}$ II region is $\sim 4.6 \times 10^{47} \mathrm{~s}^{-1}$. This could be supplied by an 09.5 ZAMS star, which has a luminosity of $3.8 \times 10^{4} L_{\odot}$. No OB association or optical H II region is seen nearby to G31.28+0.06 although W 43 is located $25^{\prime}$ south. The nature of the NVSS radio source in N-E is unclear. G31.28-MM2 and -MM3 are much weaker mm emitters. G 31.28-MM1 and G 31.28-MM2 also coincide with sub-mm and mid-IR sources within the positional accuracy (Fig. 2b). In Fig. 2c, a SED model is presented for the clumps 1 and 2. The SED of G31.28-MM1 is consistent with that of $\sim 10^{5} L_{\odot}$ and $\sim 10^{3} M_{\odot}$ clump. The SED of G 31.28-MM1 is not well modelled between 8.3 and $12.1 \mu \mathrm{m}$. This may be due to silicate absorption at $8.7 \mu \mathrm{m}$ that is not taken into account in our modelling. $\mathrm{CH}_{3} \mathrm{CN}$ and $\mathrm{C}^{18} \mathrm{O}$ lines (Fig. 2d) were detected toward the methanol maser site at velocities falling in the methanol maser velocity range (106 to $114 \mathrm{~km} \mathrm{~s}^{-1}$ ). The gas column density and temperature agree quite well with those derived from dust observations. The clump is gravitationally bound (see Table 5). G 31.28-MM2 is less luminous $\left(10^{3} L_{\odot}\right)$, less massive $\left(340 M_{\odot}\right)$ and much cooler (16 K) than G 31.28-MM1.

\subsection{2. $\mathrm{G} 59.78+0.06$}

G59.78+0.06 is a typical isolated maser, with no bright UC H II region associated with it (Fig. 3). The maser site, however, coincides with an IRAS source satisfying the Wood \& Churchwell criteria for a UC H II region, as well as a weak radio source of flux $\sim 1 \mathrm{mJy}$ (Sridharan et al. 2002). The 1.2-mm continuum map reveals a "peanut" shape with two clumps, G 59.78-MM1 and -MM2. G 59.78-MM1 is the coolest clump with no mid-IR emission. Since SCUBA observations could not be made, the SED could not be drawn. In contrast, the methanol maser site G59.78-MM2 is a much warmer clump of gas and dust. IRAS data was used in compiling the SED for G 59.78-MM2, given the close spatial coincidence between all types of emission. A luminosity of $\sim 10^{4} L_{\odot}$ and a mass of $\sim 10^{2} M_{\odot}$ were estimated for a distance of $2.1 \mathrm{kpc}$. Strong $\mathrm{C}^{18} \mathrm{O}$ emission was also detected whereas the $\mathrm{CH}_{3} \mathrm{CN}$ lines are weak and thus only lower limits are given in Table $5 . \mathrm{CH}_{3} \mathrm{CN}$ and $\mathrm{C}^{18} \mathrm{O}$ lines were detected toward the methanol maser site at velocities falling in the methanol maser velocity range (16 to $27 \mathrm{~km} \mathrm{~s}^{-1}$ ). G 59.78-MM2 is gravitationally bound within the mass estimate errors. 

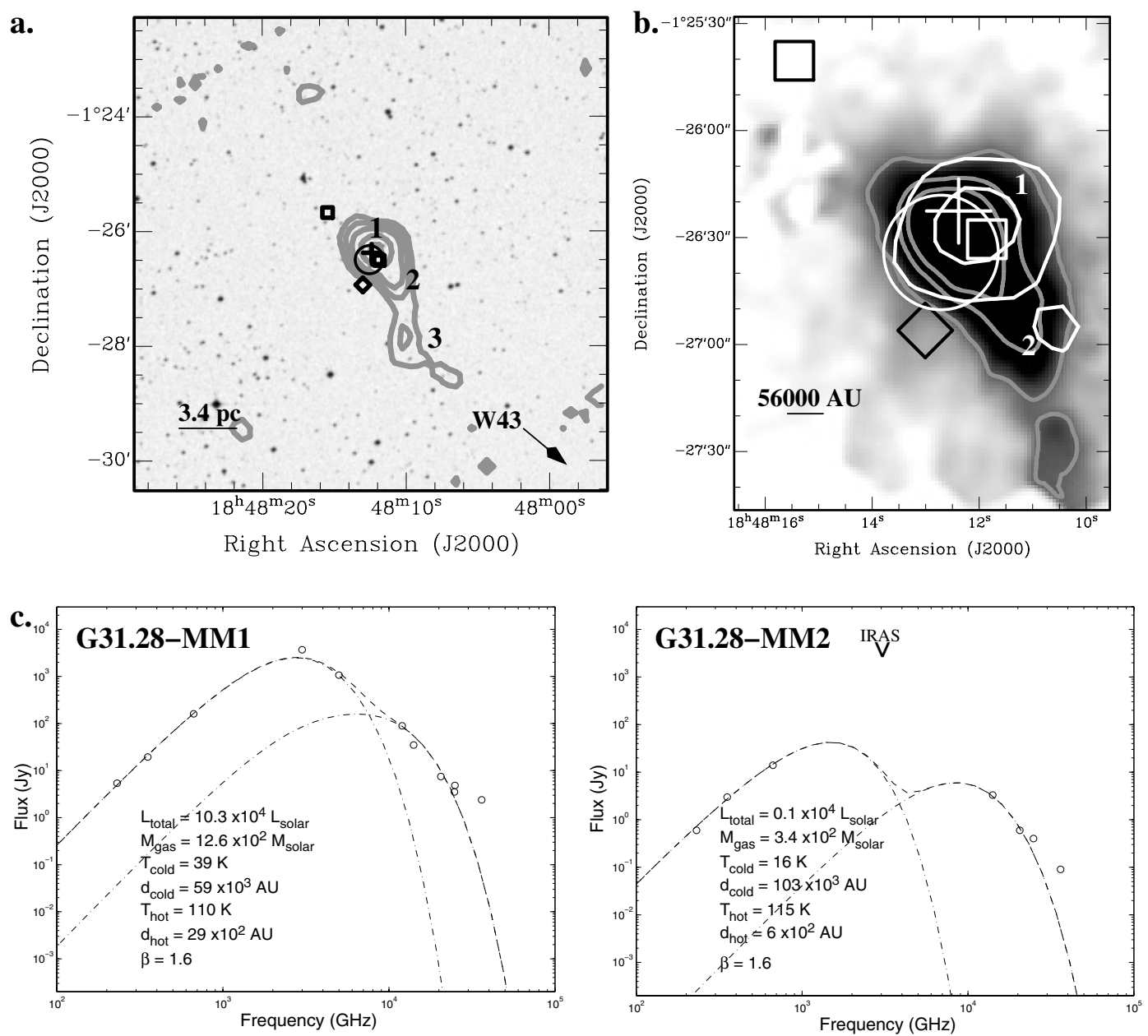

d.
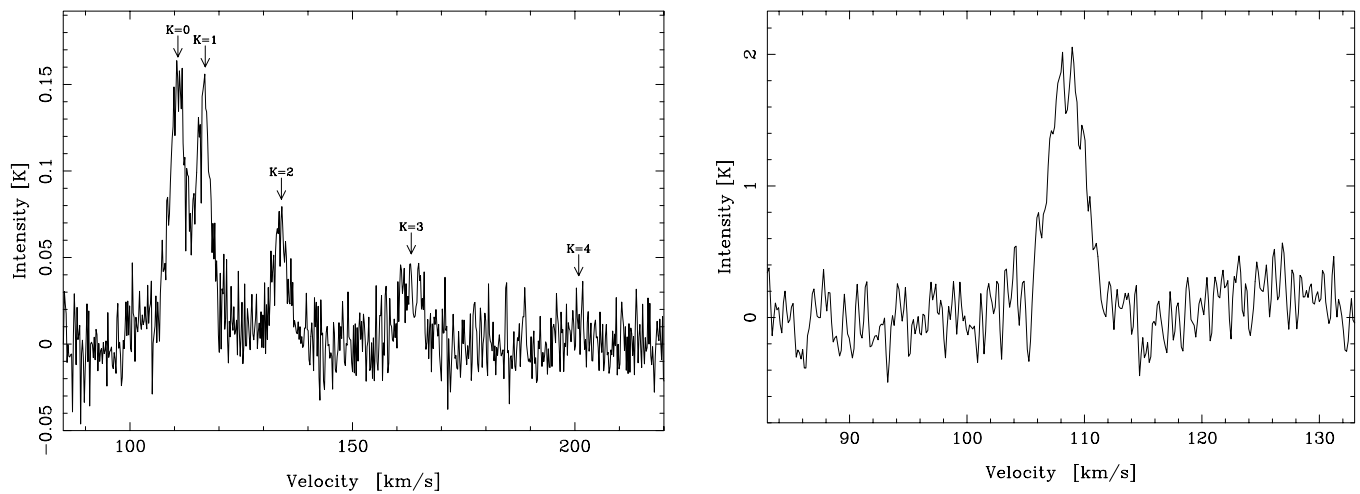

Fig. 2. G 31.28+0.06. a) Optical image (grey scale) of the neighbourhood of G31.28+0.06. The grey contours represent the 1.2-mm continuum emission imaged by SIMBA $(5,10,20,50$ and $90 \%$ of peak flux). The cross represents the position of the methanol masers, the squares are the radio continuum sites, the diamond is a recombination line site and the large circle shows the position of the IRAS source. The methanol maser source is not coincident with any visible object or optically visible HII region but it is associated with a strong mm source. b) Close-up of the mm sources 1 and 2. 850- $\mu \mathrm{m}$ SCUBA (grey scale), 450- $\mu \mathrm{m}$ SCUBA (grey contours and 10, 50 and 90\% of peak flux) and 21- $\mu \mathrm{m}$ MSX (white contours and 20 and $60 \%$ of peak flux) images. The mm sources 1 and 2 are also seen with SCUBA and MSX. c) SEDs of the mm sources 1 and 2. The IRAS icon shows the IRAS $100-\mu \mathrm{m}$ flux level. d) $\mathrm{CH}_{3} \mathrm{CN}$ and $\mathrm{C}^{18} \mathrm{O}$ line spectra.

\subsubsection{G $173.49+2.42(\mathrm{~S} 231, \mathrm{~S} 233 \mathrm{IR})$}

G 173.49+2.42, also known as S231 in maser catalogues (e.g. Pestalozzi et al. 2004) or as S233IR (e.g. Porras et al. 2000), is a methanol maser source without any associated visible starforming region. The methanol maser site is surrounded by three H II regions (Israel \& Felli 1978), S231 (north), S233 (north-west) and S235 (east), which are too offset to be seen in Fig. 4a. Two radio sources are observed east from the maser site (Fig. 4a) and are associated with source S235 \#2 in Israel \& Felli (1978). No radio continuum detection has been reported toward G 173.49+2.42 in Sridharan et al. (2002). 

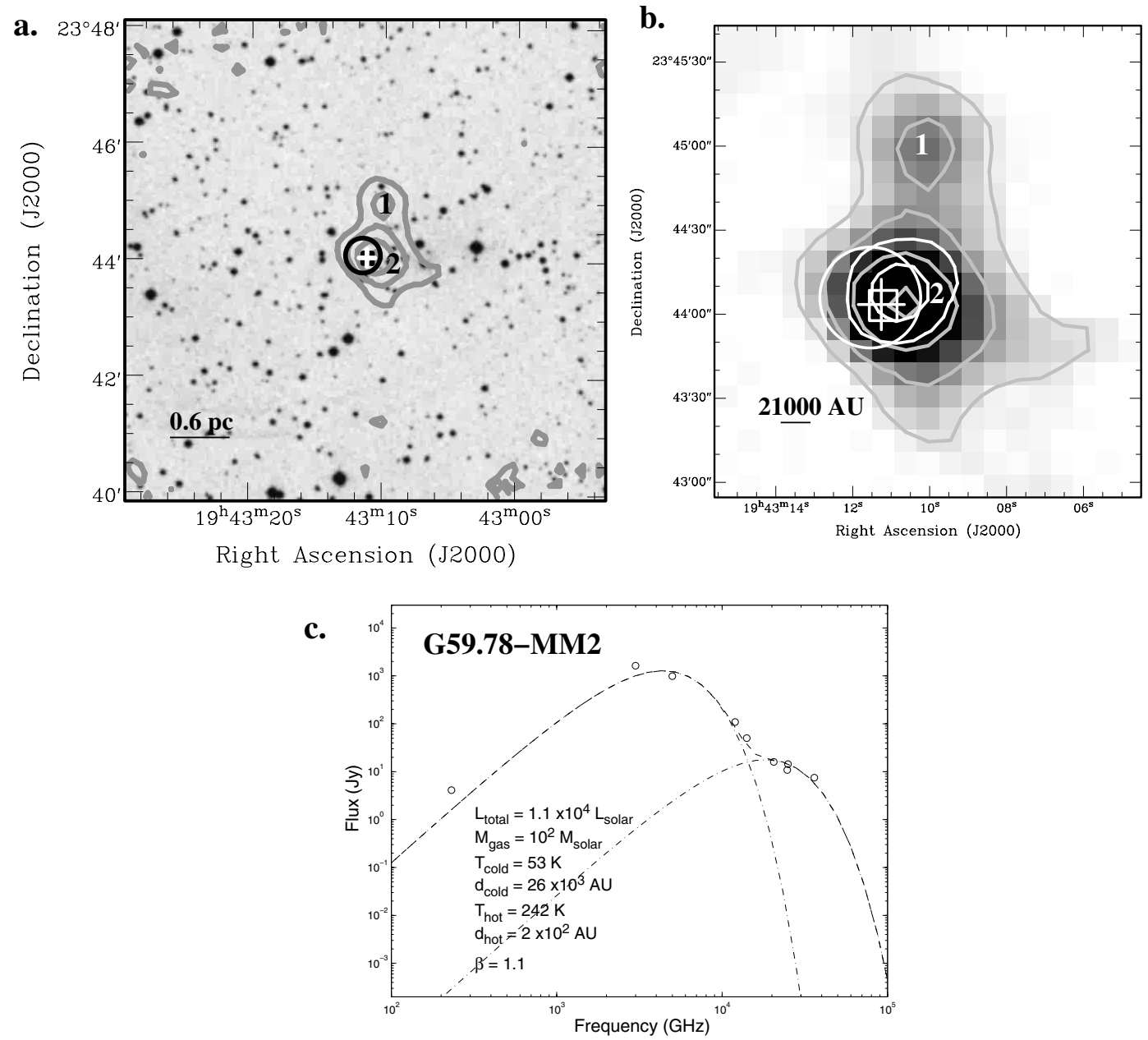

d.
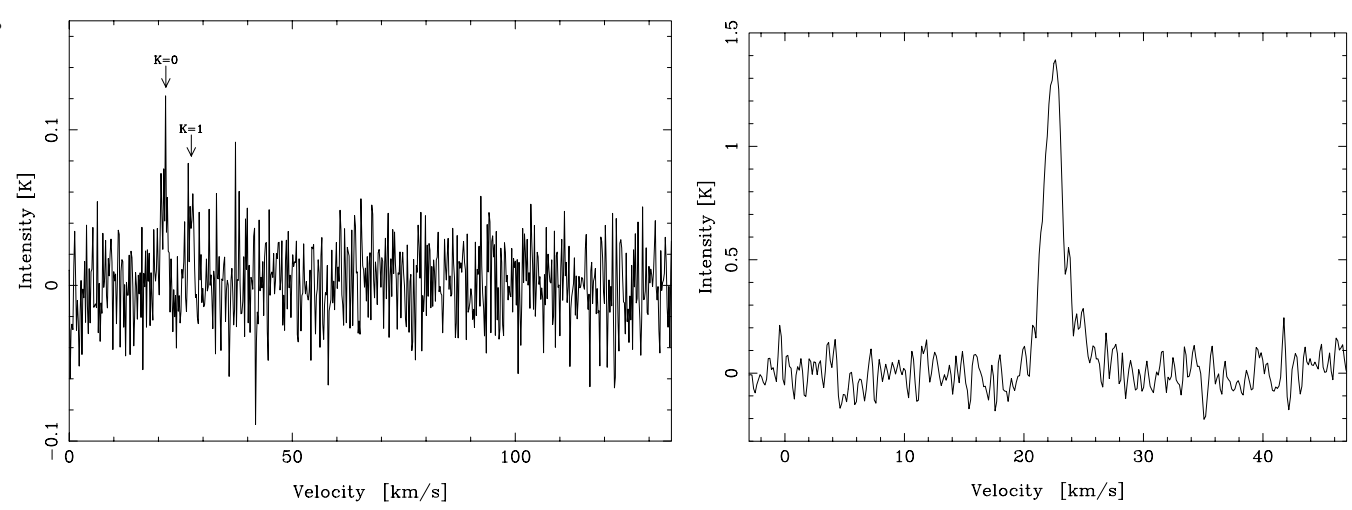

Fig. 3. G 59.78+0.06. a) Optical image (grey scale) of the neighbourhood of G 59.78+0.06. The grey contours represent the 1.2-mm continuum emission imaged by SIMBA (10, 20, 50 and $90 \%$ of peak flux). The cross represents the position of the methanol masers, the squares are the radio continuum sites and the large circle shows the position of the IRAS source. The methanol maser source is not coincident with any visible object or optically visible HII region but it is associated with a strong mm source. b) Close-up of the mm sources 1 and 2. 1.2-mm SIMBA (grey scale and contours; 10, 20, 50 and $90 \%$ of peak flux) and 21- $\mu \mathrm{m}$ MSX (white contours; 20 and $60 \%$ of peak flux) images. The mm source 2 is also seen with MSX. c) SED of the mm source 2. d) $\mathrm{CH}_{3} \mathrm{CN}$ and $\mathrm{C}^{18} \mathrm{O}$ line spectra.

In contrast, the sub-mm continuum map is rich in emission sites. Four objects were detected with SCUBA: G 173.49MM1, G 173.49-MM2, G 173.49-MM3 and G 173.49-MM4. G 173.49-MM1 harbours the methanol maser, exhibits midIR emission and coincides with one of the 1.2-mm continuum sources observed by Beuther et al. (2002). G 173.49-MM2 and G 173.49-MM3 are only seen at sub- $\mathrm{mm} / \mathrm{mm}$ wavelengths. G 173.49-MM4 appears to be associated with the far-IR source reported in the IRAS archives. The four mm objects are also associated with $\mathrm{H}_{2}$ nebulosities (Porras et al. 2000). SCUBA observations of G 173.49-MM3 are incomplete because it appears at the edge of the map. SEDs are built for G 173.49-MM1, 

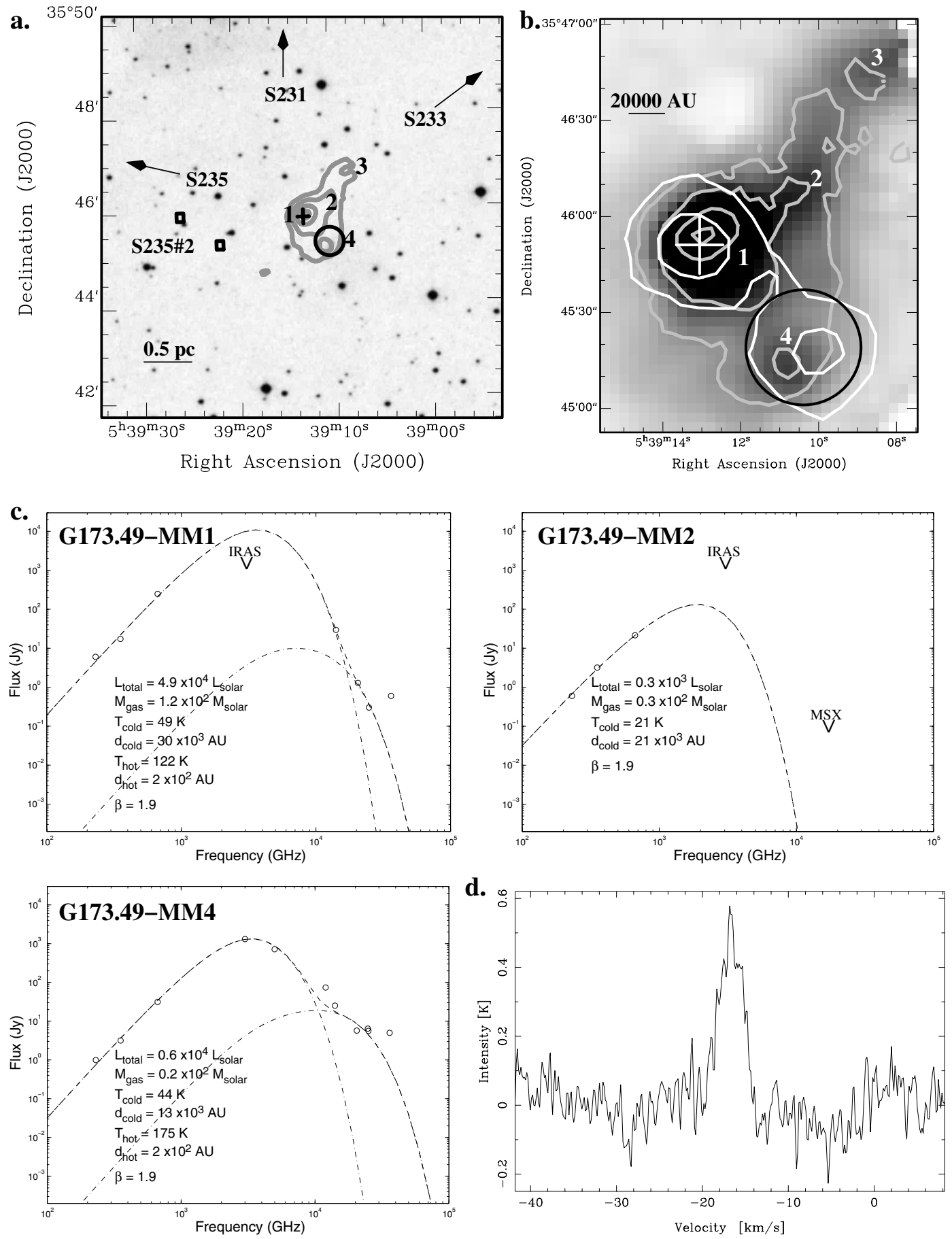

Fig. 4. G 173.49+2.42 (S231). a) Optical image (grey scale) of the neighbourhood of G $173.49+2.42$. The grey contours represent the $850-\mu \mathrm{m}$ continuum emission imaged by SCUBA (10, 20, 50 and $90 \%$ of peak flux). Note that the SCUBA map does not entirely cover the optical map. The cross represents the position of the methanol masers, the squares are the radio continuum sites and the large circle shows the position of the IRAS source. The methanol maser source is not coincident with any visible object or optically visible HII region but it is associated with a strong mm source. b) Close-up of the mm sources 1, 2, 3 and 4. 850- $\mu \mathrm{m}$ SCUBA (grey scale), 450- $\mu \mathrm{m}$ SCUBA (grey contours; 10, 20, 50 and $90 \%$ of peak flux) and 21- $\mu$ m MSX (white contours; 20 and 60\% of peak flux) images. The mm sources are also seen with SCUBA, and MM1 and MM4 are seen with MSX. c) SEDs of the mm sources 1, 2 and 4. The IRAS and MSX icons show the IRAS 100- $\mu \mathrm{m}$ flux level and MSX 20- $\mu \mathrm{m}$ image noise rms level. d) $\mathrm{C}^{18} \mathrm{O}$ line spectrum.

G 173.49-MM2 and G 173.49-MM4, with only a single greybody function fitted to the observed data of G173.49-MM2. The single grey-body fit is certainly a rough estimate but it confirms that G 173.49-MM2 is the coolest clump. G 173.49-MM1 and G 173.49-MM4 are modelled as massive $\left(>20 M_{\odot}\right)$ and luminous $\left(>10^{3} L_{\odot}\right)$ clumps for a distance of $1.8 \mathrm{kpc}$. The SED of G 173.49-MM1 is not well modelled between 8.3 and $12.1 \mu \mathrm{m}$. This may be due to silicate absorption at $8.7 \mu \mathrm{m}$ 

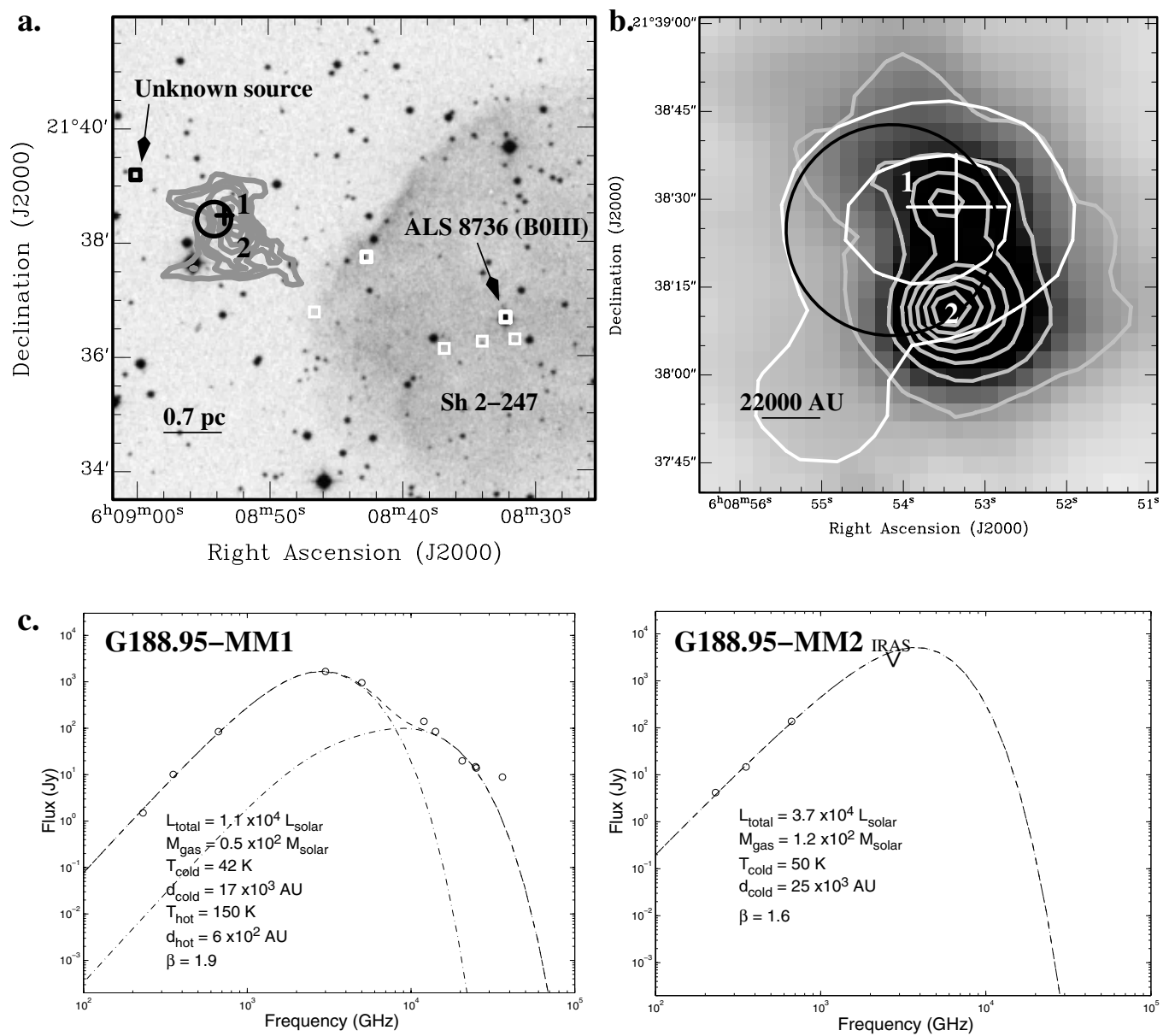

d.
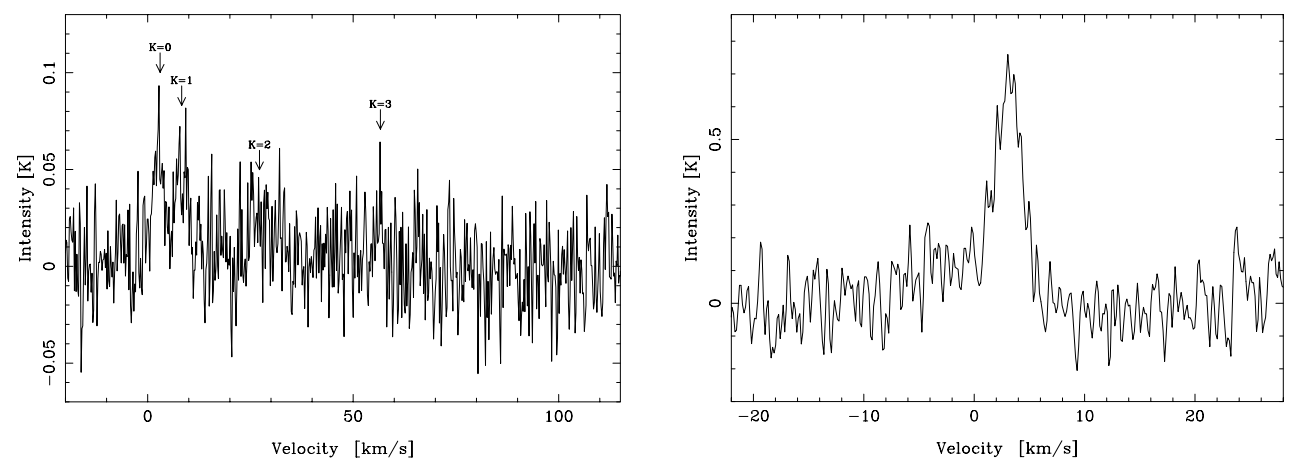

Fig. 5. G 188.95+0.89 (AFGL 5180). a) Optical image (grey scale) of the neighbourhood of G 188.95+0.89. The grey contours represent the 1.2-mm continuum emission imaged by SIMBA $(10,20,30,50.70$ and $90 \%$ of peak flux). The cross represents the position of the methanol masers, the squares are the radio continuum sites and the large circle shows the position of the IRAS source. The methanol maser source is not coincident with any visible object or optically visible HII region but it is associated with a strong mm source. b) Close-up of the mm sources 1 and 2. 850- $\mu \mathrm{m}$ SCUBA (grey scale), 450- $\mu \mathrm{m}$ SCUBA (grey contours; 30, 40, 50, 60, 7080 and 90\% of peak flux) and 21- $\mu \mathrm{m}$ MSX (white contours; 20 and $60 \%$ of peak flux) images. The mm sources 1 and 2 are also seen with SCUBA and MSX. c) SEDs of mm sources 1 and 2. The IRAS icon shows the IRAS $100-\mu$ m flux level. d) $\mathrm{CH}_{3} \mathrm{CN}$ and $\mathrm{C}^{18} \mathrm{O}$ line spectra.

that is not taken into account in our modelling. $\mathrm{CH}_{3} \mathrm{CN}$ and $\mathrm{C}^{18} \mathrm{O}$ lines were detected toward the methanol maser site (see Tables 3 and 4) at velocities slightly blueshifted with respect to the methanol maser velocity range $\left(-15\right.$ to $\left.-11 \mathrm{~km} \mathrm{~s}^{-1}\right)$. Column densities toward G 173.49-MM1 derived from gas and dust observations agree within a factor of 2 (see Table 5). G 173.49-MM1 is gravitationally bound within the mass estimate errors.

\subsubsection{G 188.95+0.89 (S252, AFGL 5180)}

G 188.95+0.89 is a methanol maser site seen in projection nearby Sh 2-247, a complex of H II regions and OB stars (Fig. 5a). Another radio source (G 188.95+0.91) to the east is an unknown and unresolved nebula (Kurtz et al. 1994). No radio source has been reported near the location of the maser site. 1.2-mm continuum emission is detected toward the methanol 

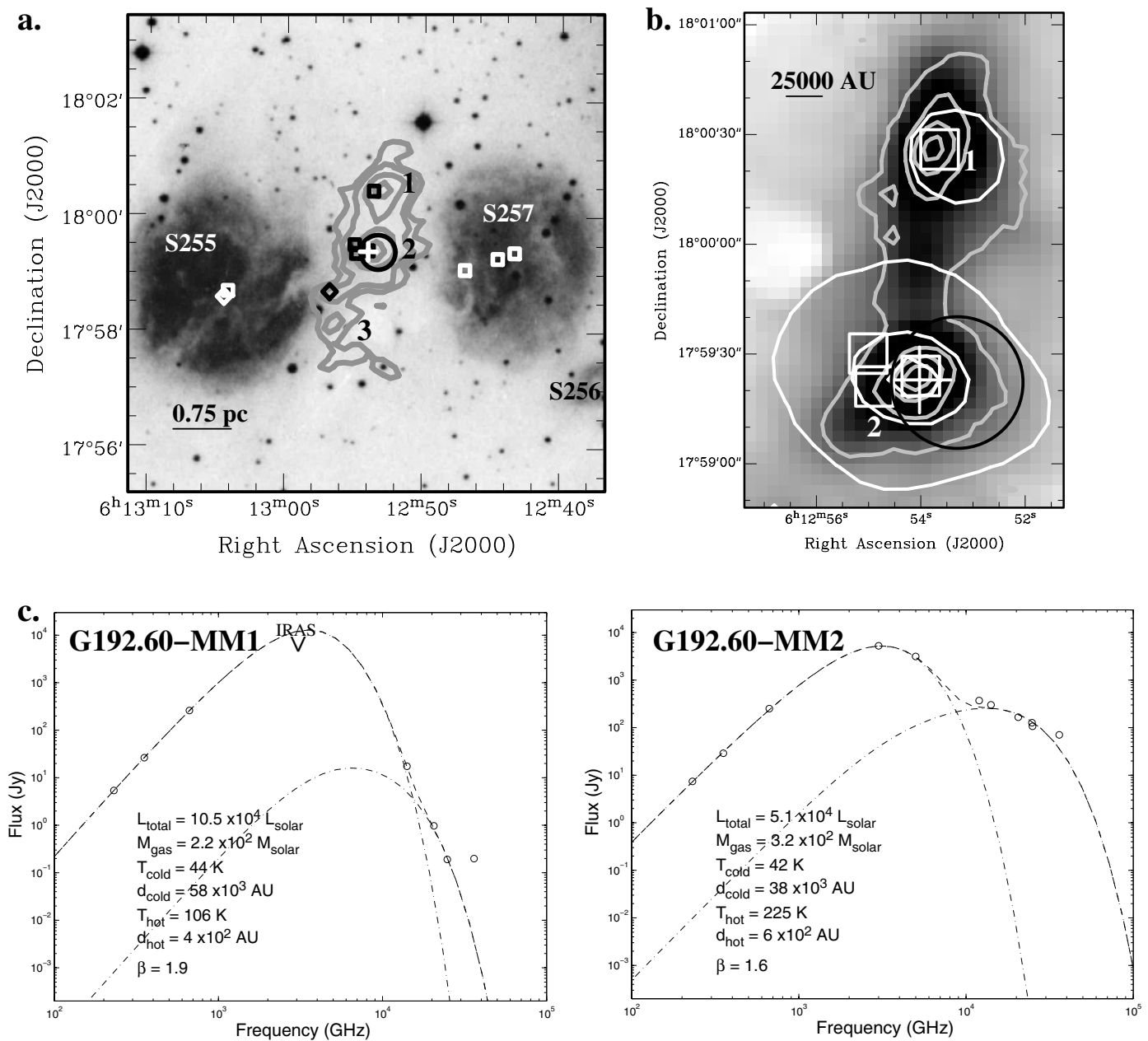

d.
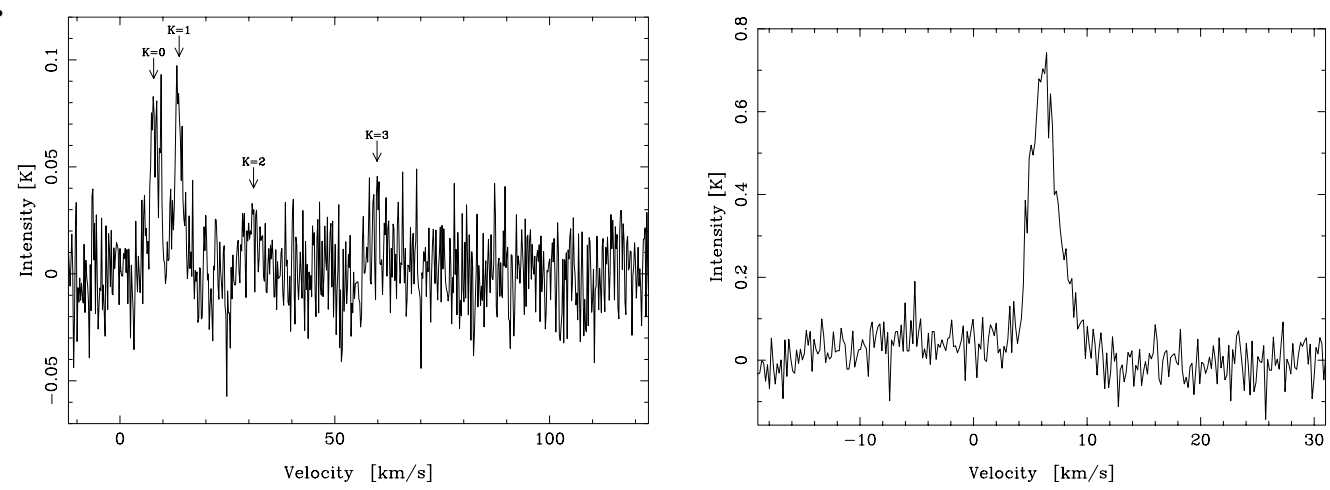

Fig. 6. G 192.60-0.05 (S255IR). a) Optical image (grey scale) of the neighbourhood of G 192.60-0.05. The grey contours represent the 1.2-mm continuum emission imaged by SIMBA (10,20, 50 and $90 \%$ of peak flux). The cross represents the position of the methanol masers, the squares are the radio continuum sites, the diamond is a recombination line site and the large circle shows the position of the IRAS source. The methanol maser source is not coincident with any visible object or optically visible HII region but it is associated with a strong mm source. b) Close-up of the mm sources 1 and 2. 850- $\mu \mathrm{m}$ SCUBA (grey scale), 450- $\mu \mathrm{m}$ SCUBA (grey contours; 25, 45, 65 and 85\% of peak flux) and 21- $\mu \mathrm{m}$ MSX (white contours; 5, 45 and $85 \%$ of peak flux) images. The mm sources 1 and 2 are also seen with SCUBA and MSX. c) SEDs of mm sources 1 and 2. The IRAS icon shows the IRAS $100-\mu$ m flux level. d) $\mathrm{CH}_{3} \mathrm{CN}$ and $\mathrm{C}^{18} \mathrm{O}$ line spectra.

maser position. The mm map is divided into two clumps, G 188.95-MM1 and -MM2. At a distance of $2.2 \mathrm{kpc}$ they are just resolved with the beam of SIMBA/SEST. G 188.95-MM1 is the methanol maser site and exhibits emission from midIR to mm. G 188.95-MM2 is also a strong sub-mm and mm source, but does not coincide with any mid-IR peak emission.
A third region to the south-east appears in the MSX contour map. The far-IR emission observed with IRAS probably includes emission from both G 188.95-MM1 and -MM2. The SED of G 188.95-MM1 requires a large mass $\left(50 M_{\odot}\right)$ and high luminosity $\left(\sim 1.1 \times 10^{4} L_{\odot}\right)$. The SED of G $188.95-M M 1$ is not well modelled between 8.3 and $12.1 \mu \mathrm{m}$. This may be due to 
Table 1. Continuum fluxes. Positions are the 1.2-mm peak emission positions. Mid-IR fluxes are from MSX images. Sub-mm fluxes from SCUBA images. Mm fluxes from SIMBA images. “-” means that no emission was detected. Notes: * fluxes interpolated from SED diagrams; ${ }^{* *}$ MAMBO mm fluxes from Beuther et al. (2002). References: ${ }^{1}$ Walsh et al. (1998); ${ }^{2}$ Kurtz et al. (1994); ${ }^{3}$ Sridharan et al. (2002); ${ }^{4}$ Snell \& Bally (1986).

\begin{tabular}{|c|c|c|c|c|c|c|c|c|c|c|}
\hline \multirow[b]{2}{*}{ Source } & \multicolumn{2}{|c|}{ Position } & \multicolumn{4}{|c|}{ Flux (Jy) Mid-IR } & \multicolumn{2}{|c|}{ Sub-mm } & \multirow{2}{*}{$\begin{array}{l}\mathrm{Mm} \\
1.2 \\
(\mathrm{~mm})\end{array}$} & \multirow{2}{*}{$\begin{array}{l}\text { Radio } \\
\text { (mJy) }\end{array}$} \\
\hline & $\begin{array}{l}\text { RA } \\
(\mathrm{J} 2000)\end{array}$ & $\begin{array}{l}\text { Dec } \\
(\mathrm{J} 2000)\end{array}$ & $\begin{array}{l}8.3 \\
(\mu \mathrm{m})\end{array}$ & $\begin{array}{l}12.1 \\
(\mu \mathrm{m})\end{array}$ & $\begin{array}{l}14.6 \\
(\mu \mathrm{m})\end{array}$ & $\begin{array}{l}21.3 \\
(\mu \mathrm{m})\end{array}$ & $\begin{array}{l}450 \\
(\mu \mathrm{m})\end{array}$ & $\begin{array}{l}850 \\
(\mu \mathrm{m}) \\
\end{array}$ & & \\
\hline G 31.28-MM1 & 184812.1 & -012620 & 2.4 & 3.5 & 7.5 & 34.9 & 160.0 & 19.5 & 5.4 & $80(8.6 \mathrm{GHz})^{1,2}$ \\
\hline G 31.28-MM2 & 184810.8 & -012654 & 0.1 & 0.4 & 0.6 & 3.3 & 14.0 & 3.0 & 0.6 & $<0.4(15 \mathrm{GHz})^{2}$ \\
\hline G 31.28-MM3 & 184810.0 & -012755 & - & - & - & - & - & - & 0.3 & $<0.4(15 \mathrm{GHz})^{2}$ \\
\hline G 59.78-MM1 & 194310.0 & 234459 & - & - & - & - & - & - & 0.9 & $<1(8.6 \mathrm{GHz})$ \\
\hline G 59.78-MM2 & 194310.6 & 234403 & 7.5 & 10.9 & 16.0 & 50.1 & $35.8^{*}$ & $5.8^{*}$ & 4.1 & $1(8.6 \mathrm{GHz})^{3}$ \\
\hline G 173.49-MM1 & 053913.0 & 354554 & 0.6 & 0.3 & 1.3 & 29.8 & 249.4 & 17.3 & $6.0^{* *}$ & $<1(8.6 \mathrm{GHz})^{3}$ \\
\hline G 173.49-MM2 & 053910.8 & 354606 & - & - & - & - & 21.3 & 3.2 & $0.6^{* *}$ & $<1(8.6 \mathrm{GHz})^{3}$ \\
\hline G 173.49-MM3 & 053908.1 & 354648 & - & - & - & - & - & 2.0 & $0.4^{* *}$ & $<1(8.6 \mathrm{GHz})^{3}$ \\
\hline G 173.49-MM4 & 053910.8 & 354512 & 5.0 & 6.4 & 5.7 & 25.3 & 31.2 & 3.2 & $1.0^{* *}$ & $<1(8.6 \mathrm{GHz})^{3}$ \\
\hline G 188.95-MM1 & 060852.8 & 213837 & 8.9 & 14.8 & 19.7 & 85.2 & 83.9 & 10.2 & 1.5 & $<0.4(15 \mathrm{GHz})^{2}$ \\
\hline G 188.95-MM2 & 060852.2 & 213813 & - & - & $0.1^{*}$ & $17.6^{*}$ & 136.6 & 14.6 & 4.2 & $<0.4(15 \mathrm{GHz})^{2}$ \\
\hline G 192.60-MM1 & 061252.9 & 180027 & 0.2 & 0.2 & 1.0 & 17.4 & 260.0 & 26.3 & 5.4 & $22(8.6 \mathrm{GHz})^{2}$ \\
\hline G 192.60-MM2 & 061253.4 & 175923 & 70.6 & 126.5 & 165.4 & 301.4 & 250.0 & 28.9 & 7.4 & $3-5(5 \mathrm{GHz})^{4}$ \\
\hline G 192.60-MM3 & 061256.8 & 175803 & - & - & - & - & - & - & 0.8 & $<0.4(15 \mathrm{GHz})^{2}$ \\
\hline
\end{tabular}

Table 2. IRAS fluxes and association with mm sources.

\begin{tabular}{lllllllll}
\hline \hline Source & IRAS name & $\begin{array}{l}12 \\
(\mu \mathrm{m})\end{array}$ & $\begin{array}{l}25 \\
(\mu \mathrm{m})\end{array}$ & $\begin{array}{l}60 \\
(\mu \mathrm{m})\end{array}$ & $\begin{array}{l}100 \\
(\mu \mathrm{m})\end{array}$ & $\log \left(F_{25} / F_{12}\right)$ & $\log \left(F_{60} / F_{12}\right)$ & Association \\
\hline G 31.28+0.06 & $18456-0129$ & 4.85 & 89.33 & 1071.0 & 3693.0 & 1.26 & 2.34 & G 31.28-MM1 \\
G 59.78+0.06 & $19410+2336$ & 14.4 & 108.8 & 982.5 & 1631.0 & 0.98 & 1.83 & G 59.78-MM2 \\
G 173.49+2.42 & $05358+3543$ & 5.6 & 74.7 & 722.3 & 1310.0 & 1.12 & 2.11 & G 173.49-MM4 \\
G 188.95+0.89 & $06058+2138$ & 13.9 & 140.2 & 955.5 & 1666.0 & 1.00 & 1.83 & G 188.95-MM1 \\
G 192.60-0.05 & $06099+1800$ & 107.2 & 371.6 & 3145.0 & 5285.0 & 0.54 & 1.47 & G 192.60-MM2 \\
\hline
\end{tabular}

Table 3. $\mathrm{CH}_{3} \mathrm{CN}$ Spectral line parameters for the methanol maser sites derived from Gaussian fitting.

\begin{tabular}{lllllll}
\hline \hline Source & $K$ & $\begin{array}{l}v_{\text {lsr }} \\
\left(\mathrm{km} \mathrm{s}^{-1}\right)\end{array}$ & $\begin{array}{l}T_{A}^{*} \\
(\mathrm{~K})\end{array}$ & $\begin{array}{l}\mathrm{rms} \\
(\mathrm{K})\end{array}$ & $\begin{array}{l}\Delta v \\
\left(\mathrm{~km} \mathrm{~s}^{-1}\right)\end{array}$ & $\begin{array}{l}\int T_{A}^{*} \mathrm{~d} v \\
\left(\mathrm{~K} \mathrm{~km} \mathrm{~s}^{-1}\right)\end{array}$ \\
\hline G 31.28-MM1 & 0 & 110.5 & 0.15 & 0.01 & 4.1 & 0.66 \\
& 1 & 110.5 & 0.13 & 0.01 & 4.1 & 0.59 \\
& 2 & 110.5 & 0.06 & 0.01 & 4.0 & 0.24 \\
& 3 & 110.9 & 0.03 & 0.01 & 4.0 & 0.14 \\
G 59.78-MM2 & 4 & 110.0 & 0.01 & 0.01 & 4.1 & 0.01 \\
& 1 & 21.3 & 0.07 & 0.02 & 1.8 & 0.14 \\
G 173.49-MM1 & 2 & 21.5 & 0.05 & 0.02 & 1.5 & 0.09 \\
G 188.95-MM1 & 0 & see & Kalenskii & et & al. & $(2000)$ \\
& 1 & 2.3 & 0.05 & 0.02 & 4.0 & 0.23 \\
& 2 & 2.5 & 0.04 & 0.02 & 4.1 & 0.21 \\
G 192.60-MM2 & 3 & 3.8 & 0.03 & 0.02 & 4.1 & 0.13 \\
& 0 & 8.2 & 0.07 & 0.02 & 4.1 & 0.10 \\
& 1 & 7.8 & 0.08 & 0.02 & 3.0 & 0.24 \\
& 2 & 7.8 & 0.03 & 0.02 & 2.7 & 0.22 \\
& 3 & 7.6 & 0.03 & 0.02 & 3.1 & 0.12 \\
\hline
\end{tabular}

silicate absorption at $8.7 \mu \mathrm{m}$ that is not taken into account in our modelling. Similarly, the SED of G 188.95-MM2, modelled with a single grey-body function, also requires a high mass and luminosity. Note that a total mass of $60 M_{\odot}$ is given in Lada \& Lada (2003) for AFGL 5180 at a distance of $1.5 \mathrm{kpc} .170 M_{\odot}$ in total are derived from SIMBA observations for G 188.95+0.89 at a distance of $2.2 \mathrm{kpc}$. By taking $1.5 \mathrm{kpc}$ instead of $2.2 \mathrm{kpc}$ in Eq. (1), a total mass of $78 M_{\odot}$ would have been calculated. Our result is consistent with the Lada \& Lada's mass estimate. $\mathrm{CH}_{3} \mathrm{CN}$ and $\mathrm{C}^{18} \mathrm{O}$ lines were detected toward the methanol maser site (see Tables 3 and 4) at velocities blueshifted with respect to the methanol maser velocity range ( 8 to $12 \mathrm{~km} \mathrm{~s}^{-1}$ ). 
Table 4. $\mathrm{C}^{18} \mathrm{O}$ spectral line parameters for the methanol maser sites derived from Gaussian fitting.

\begin{tabular}{lllllll}
\hline \hline Source & $\begin{array}{l}v_{\mathrm{lsr}} \\
\left(\mathrm{km} \mathrm{s}^{-1}\right)\end{array}$ & $\begin{array}{l}T_{A}^{*} \\
(\mathrm{~K})\end{array}$ & $\begin{array}{l}\mathrm{rms} \\
(\mathrm{K})\end{array}$ & $\begin{array}{l}\Delta v \\
\left(\mathrm{~km} \mathrm{~s}^{-1}\right)\end{array}$ & $\begin{array}{l}\int T_{A}^{*} \mathrm{~d} v \\
\left(\mathrm{~K} \mathrm{~km} \mathrm{~s}^{-1}\right)\end{array}$ & $\begin{array}{l}\Delta v_{\mathrm{CH}_{3} \mathrm{OH}} \\
\left(\mathrm{km} \mathrm{s}^{-1}\right)\end{array}$ \\
\hline G 31.28-MM1 & 108.5 & 1.89 & 0.17 & 3.6 & 7.20 & $106 ; 114$ \\
G 59.78-MM2 & 22.5 & 1.31 & 0.07 & 2.0 & 2.83 & $16 ; 27$ \\
G 173.49-MM1 & -16.7 & 0.51 & 0.06 & 3.2 & 1.71 & $-15 ;-11$ \\
G 188.95-MM1 & 3.0 & 0.66 & 0.08 & 3.2 & 2.25 & $8 ; 12$ \\
G 192.60-MM2 & 6.2 & 0.67 & 0.04 & 3.0 & 2.15 & $1 ; 6$ \\
\hline
\end{tabular}

Table 5. Physical properties of the methanol maser sites derived from the SED and spectral line analysis. $N_{\mathrm{H}_{2}}$ and $n_{\mathrm{H}_{2}}$ are derived from $M_{\mathrm{gas}}$ in the continuum case by using an angular size of $24^{\prime \prime}$. The values of $N_{\mathrm{H}_{2}}$ derived using $d_{\text {cold }}$ are given in parentheses. $M_{\mathrm{gas}}$ in the spectral line case is derived from $N_{\mathrm{H}_{2}}$ by using an angular size of $34^{\prime \prime}$.

\begin{tabular}{|c|c|c|c|c|c|c|c|c|c|c|}
\hline \multirow[b]{2}{*}{ Source } & \multicolumn{5}{|c|}{ Continuum } & \multicolumn{5}{|c|}{ Spectral line } \\
\hline & $\begin{array}{l}T_{\text {cold }} \\
(\mathrm{K})\end{array}$ & $\begin{array}{l}M_{\text {gas }} \\
\left(10^{2} M_{\odot}\right)\end{array}$ & $\begin{array}{l}N_{\mathrm{H}_{2}} \\
\left(10^{22} \mathrm{~cm}^{-2}\right)\end{array}$ & $\begin{array}{l}n_{\mathrm{H}_{2}} \\
\left(10^{4} \mathrm{~cm}^{-3}\right)\end{array}$ & $\begin{array}{l}L_{\text {total }} \\
\left(10^{4} L_{\odot}\right)\end{array}$ & $\begin{array}{l}T_{\text {rot }} \\
(\mathrm{K})\end{array}$ & $\begin{array}{l}M_{\text {gas }} \\
\left(10^{2} M_{\odot}\right)\end{array}$ & $\begin{array}{l}N_{\mathrm{H}_{2}} \\
\left(10^{22} \mathrm{~cm}^{-2}\right)\end{array}$ & $\begin{array}{l}n_{\mathrm{H}_{2}} \\
\left(10^{4} \mathrm{~cm}^{-3}\right)\end{array}$ & $\begin{array}{l}M_{\text {vir }} \\
\left(10^{2} M_{\odot}\right)\end{array}$ \\
\hline G 31.28-MM1 & 39 & 12.6 & $17.5(90.3)$ & 13.1 & 10.3 & 34 & 21.6 & 15.1 & 8.0 & 7.4 \\
\hline G 59.78-MM2 & 53 & 1.0 & $9.8(36.5)$ & 19.5 & 1.1 & $>18$ & $>0.8$ & $>3.8$ & $>5.4$ & 0.9 \\
\hline G 173.49-MM1 & 49 & 1.2 & $15.5(31.8)$ & 36.0 & 4.9 & 69 & 1.0 & 6.5 & 10.8 & 1.8 \\
\hline G 188.95-MM1 & 42 & 0.5 & $4.5(41.0)$ & 8.6 & 1.1 & 58 & 1.6 & 7.4 & 10.0 & 2.3 \\
\hline G 192.60-MM2 & 42 & 3.2 & $22.3(59.5)$ & 37.3 & 5.1 & 55 & 1.5 & 5.3 & 6.3 & 2.4 \\
\hline
\end{tabular}

Similar column densities are derived from gas and dust emission. The gravitational status of G 188.95-MM1 is unclear.

\subsubsection{G 192.60-0.05 (S255IR)}

G 192.60-0.05 is a methanol maser site situated between two H II regions, S255 and S257 (see Minier et al. 2001 and references therein), consisting of OB stars (Fig. 6a). SIMBA observations at $1.2 \mathrm{~mm}$ reveal a dusty filament elongated northsouth, centred at the location of the methanol maser. Three $\mathrm{mm}$ sources are identified: G 192.60-MM1, G 192.60-MM2 and G 192.60-MM3. An additional mm core might be present at a median position between G 192.60-MM1 and G 192.60-MM2 (Fig. 6b), but is too poorly resolved to be successfully fitted as a compact source. G 192.60-MM1 is also concident with a UC $\mathrm{H}$ II region, a sub-mm source, and a mid-IR source (Fig. 6b). G 192.60-MM2 is the site of the methanol maser. It also contains three weak and very confined radio sources that might be interpreted as hyper-compact H II regions (Minier et al. 2001 for explanation). It is also associated with mid-IR, far-IR and sub-mm emission. The IRAS source coincides with the maser site. G 192.60-MM3 is a colder source that is not clearly identified as a clump. G 192.60-MM1 and G 192.60-MM2 are modelled as two spherical clumps with a high mass $\left(>200 M_{\odot}\right)$ and luminosity $\left(>10^{4} L_{\odot}\right)$ for a distance of $2.6 \mathrm{kpc}$. The SED of G 192.60-MM1 is not well modelled between 8.3 and $12.1 \mu \mathrm{m}$. This may be due to silicate absorption at $8.7 \mu \mathrm{m} . \mathrm{CH}_{3} \mathrm{CN}$ and $\mathrm{C}^{18} \mathrm{O}$ lines were detected toward the methanol maser site (see Tables 3 and 4) at velocities redshifted with respect to the methanol maser velocity range $\left(1\right.$ to $\left.6 \mathrm{~km} \mathrm{~s}^{-1}\right)$. Column densities in G 192.60-MM2 derived from gas and dust observations agree within a factor 5 (see Table 5). G 192.60-MM2 is gravitationally bound within the mass estimate errors.

\subsection{Dust grain emissivity index ( $\beta$ )}

The dust grain emissivity index $\beta$ varies between 1.6 and 1.9 for all sources modelled, with the exception of G 59.78-MM2, whose SED is poorly constrained with the absence of submillimetre data. This range of values is consistent with the numerical estimates of $\beta$ presented by Ossenkopf \& Henning (1994), who found values of $\beta$ ranging between 1.8 and 2 for dust grains with ice mantles. The adopted value of $\kappa_{\mathrm{d}}=$ $0.1 \mathrm{~m}^{2} \mathrm{~kg}^{-1}$ at $\lambda=1.2 \mathrm{~mm}$ taken from Ossenkopf \& Henning (1994) is therefore self-consistent.

\section{Discussion}

\subsection{Methanol masers and MSF}

The aim of this work was to study the large-scale $(\sim 0.5-5 \mathrm{pc})$ environment of methanol masers. The results demonstrate that each radio-quiet maser site is always associated with a massive ( $>50 M_{\odot}$ ) and very luminous $\left(>10^{4} L_{\odot}\right.$ ) clump of molecular gas and dust. Whether the clumps are very young clusters, i.e. protoclusters ( $\leq \mathrm{a}$ few $10^{5} \mathrm{yr}$ ), forming high-mass stars or single protostellar cores is the subject of this discussion.

These individual clumps are at best partially resolved with the relatively low angular resolution of SIMBA, SCUBA or MSX. An estimate of their diameter is given by $d_{\text {cold }}$ in Figs. 2-6. It varies between $17 \times 10^{3}$ and $59 \times 10^{3} \mathrm{AU}$, i.e. $\sim 0.1$ to $0.3 \mathrm{pc}$. This implies $\mathrm{H}_{2}$ mass densities of $6 \times 10^{4}$ to $10^{5} M_{\odot} \mathrm{pc}^{-3}$ in the clumps with a methanol maser (Table 6). However, the uncertainties on the clump diameters is of crucial importance as the density value varies with $d_{\text {cold. }}^{3}$ By taking the beam size of $\sim 24^{\prime \prime}$ as an upper limit for each clump diameter, the mass density would be $5 \times 10^{3}$ to $3 \times$ $10^{4} M_{\odot} \mathrm{pc}^{-3}$ at heliocentric distances of 1.8 to $5.6 \mathrm{kpc}$. These values agree with those determined for star-forming clusters 
Table 6. Summary of the mm clump properties. $A_{\mathrm{V}}$ is derived from $M_{\mathrm{gas}}$ in the continuum case of Table 5. Note: * A clump angular size of $24^{\prime \prime}$ is used.

\begin{tabular}{llllll}
\hline \hline $\begin{array}{l}\text { Clump } \\
\text { name }\end{array}$ & $\begin{array}{l}D \\
(\mathrm{kpc})\end{array}$ & $\begin{array}{l}M \\
\left(10^{2} M_{\odot}\right)\end{array}$ & $\begin{array}{l}d_{\text {cold }}(\mathrm{pc}) \\
\begin{array}{l}\text { Density } \\
\left(M_{\odot} \mathrm{pc}^{-3}\right)\end{array}\end{array}$ & $(\mathrm{mag})$ \\
\hline G 31.28-MM1 & 5.6 & 12.6 & 0.3 & $89.1 \times 10^{3}$ & 186 \\
G 31.28-MM2 & 5.6 & 3.4 & 0.5 & $4.7 \times 10^{3}$ & 50 \\
G 31.28-MM3 & 5.6 & 1.8 & $0.6^{*}$ & $1.6 \times 10^{3 *}$ & 26 \\
G 59.78-MM1 & 2.1 & 0.8 & $0.2^{*}$ & $17.9 \times 10^{3 *}$ & 78 \\
G 59.78-MM2 & 2.1 & 1.0 & 0.1 & $86.9 \times 10^{3}$ & 104 \\
G 173.49-MM1 & 1.8 & 1.2 & 0.1 & $67.9 \times 10^{3}$ & 164 \\
G 173.49-MM2 & 1.8 & 0.3 & 0.1 & $49.4 \times 10^{3}$ & 40 \\
G 173.49-MM3 & 1.8 & 0.3 & $0.2^{*}$ & $5.8 \times 10^{3 *}$ & 35 \\
G 173.49-MM4 & 1.8 & 0.2 & 0.1 & $139.1 \times 10^{3}$ & 28 \\
G 188.95-MM1 & 2.2 & 0.5 & 0.1 & $95.5 \times 10^{3}$ & 48 \\
G 188.95-MM2 & 2.2 & 1.2 & 0.1 & $117.3 \times 10^{3}$ & 114 \\
G 192.60-MM1 & 2.6 & 2.2 & 0.3 & $17.2 \times 10^{3}$ & 150 \\
G 192.60-MM2 & 2.6 & 3.2 & 0.2 & $89.1 \times 10^{3}$ & 237 \\
G 192.60-MM3 & 2.6 & 1.0 & $0.3^{*}$ & $7.3 \times 10^{3 *}$ & 70 \\
\hline
\end{tabular}

such as W3:IRS5 (Megeath et al. 1996), Mon R2 (Carpenter et al. 1997), NGC 2024 in Orion B and the Trapezium (Lada $\&$ Lada 2003). More generally, our results are consistent with the physical parameters describing the least evolved embedded clusters in Lada \& Lada (2003). Embedded clusters are all associated with massive $\left(100-1000 M_{\odot}\right)$ and dense $\left(n_{\mathrm{H}_{2}}=10^{4}-10^{5} \mathrm{~cm}^{-3}\right)$ clumps with diameters $\sim 0.5-1 \mathrm{pc}$ and mass density $\sim 10^{3}-10^{4} M_{\odot} \mathrm{pc}^{-3}$ within the molecular clouds. Interestingly, the overall mass, luminosity and linear size for each star-forming complex are not much larger than those measured for protoclusters forming stars less massive than B2 stars such as L1688 in $\rho$-Oph with $L_{\text {total }}=6.7 \times 10^{3} L_{\odot}$ (due to HD 147889, a B2 star as well as the nearby Sco OB2 association), $M \sim 500 M_{\odot}$ and a diameter $\sim 1 \mathrm{pc}$ (Greene \& Young 1989; Wilking \& Lada 1983).

The visual extinction of the clumps with a methanol maser can be estimated with $A_{\mathrm{v}}=N_{\mathrm{H}_{2}} / 0.94 \times 10^{21}$ mag (Frerking et al. 1982). It varies from 40 to $240 \mathrm{mag}$, implying a high degree of embeddedness and presumably a very young age $\left(\ll 10^{6} \mathrm{yr}\right)$. In comparison, the visual extinction in the $\rho$-Oph central $\mathrm{C}^{18} \mathrm{O}$ cloud in $\mathrm{L} 1688$ is in the range $50-100 \mathrm{mag}$ (Wilking \& Lada 1983) with a column density $\sim 10^{23} \mathrm{~cm}^{-2}$ and a diameter $\sim 0.5 \mathrm{pc}$. Three $\mathrm{mm}$ cores (Oph-C, Oph-CS and Oph-E) were mapped by Motte et al. (1998) in the central cloud and have column densities ranging from $\sim 7 \times 10^{22}$ to $10^{23} \mathrm{~cm}^{-2}$, which compare to those in the methanol maser clumps $\left(4.5 \times 10^{22}\right.$ to $\left.2.2 \times 10^{23} \mathrm{~cm}^{-2}\right)$.

In summary, the methanol maser sites exhibit the properties of young, deeply embedded clusters of YSOs. Only luminosities in excess of $10^{4} L_{\odot}$ ( ZAMS B0.5, Panagia 1973) for clumps less than $\sim 1 \mathrm{pc}$ in diameter clearly suggest the presence of high-mass (>8 $M_{\odot}$ ) YSOs.

The detection of $\mathrm{CH}_{3} \mathrm{CN}$ and $\mathrm{C}^{18} \mathrm{O}$ toward each methanol maser site indicates that they are within HMCs with gas density $\geq 10^{5} \mathrm{~cm}^{-3}$. $\mathrm{CH}_{3} \mathrm{CN}$ emission usually arises from the 0.1 -pc inner part of the hot core although ground state $\mathrm{CH}_{3} \mathrm{CN}$ emission could trace a cooler region in HMCs than those probed with vibrationally excited $\mathrm{CH}_{3} \mathrm{CN}$ lines (Olmi et al. 2003). This might explain the relatively low rotational temperatures (18-69 K) in Table 5. The $\mathrm{C}^{18} \mathrm{O}$ antenna temperatures (Table 4) are much larger than those measured for $\mathrm{CH}_{3} \mathrm{CN}$ (Table 3). A possible explanation is that $\mathrm{C}^{18} \mathrm{O}$ emission arises from a larger area than $\mathrm{CH}_{3} \mathrm{CN}$ emission. The $\mathrm{CH}_{3} \mathrm{CN}$ rotational temperatures agree well with the $T_{\text {cold }}$ values (Table 5), which suggest that $\mathrm{CH}_{3} \mathrm{CN}\left(5_{K}-4_{K}\right)$ and dust emission arises from the same area. The fitted source solid angles $\left(\Omega_{\text {cold }}\right.$ and $\Omega_{\text {hot }}$ used to estimate $d_{\text {cold }}$ and $d_{\text {hot }}$ ) in the dust emission models are all smaller than the OSO and Mopra telescope beam sizes, which then probably overestimate the true size of the emitting regions. In consequence, the densities $\left(\sim 10^{5} \mathrm{~cm}^{-3}\right)$ derived from the $\mathrm{C}^{18} \mathrm{O}$ data (Table 5) with the telescope beam sizes as the source angular sizes are lower limits of the $\mathrm{CH}_{3} \mathrm{CN}$ clump densities. In addition to $\mathrm{CH}_{3} \mathrm{CN}$ emission, $\mathrm{NH}_{3}$ and $\mathrm{CH}_{3} \mathrm{OH}$ quasi-thermal lines were also detected toward G 59.78+0.06, G 173.49+2.42, G 188.95+0.89 and G192.60-0.05 (Minier \& Booth 2002). The presence of the typical HMC species $\left(\mathrm{CH}_{3} \mathrm{CN}, \mathrm{NH}_{3}\right.$ and $\mathrm{CH}_{3} \mathrm{OH}$ ) suggest again a very young age ( $\leq \mathrm{a}$ few $10^{5} \mathrm{yr}$ ) for the methanol maser sites (e.g. Rodgers \& Charnley 2001).

In conclusion, methanol masers trace very luminous, deeply embedded, massive, molecular clumps in the five starforming regions considered here. In a few of the clumps (G 31.28-MM1, G 192.60-MM2 and G 59.78-MM2), ionised sources were also detected in the radio. This implies that highmass stars are currently forming within these clumps and that the deeply embedded star-forming clumps are protoclusters of high-mass YSOs.

Yet, whether methanol masers are themselves directly associated with a massive YSO remains unclear. In G 31.28+0.06, the maser is offset by $3.5 \times 10^{4} \mathrm{AU}$ from the $\mathrm{UC} \mathrm{H}$ II region (Walsh et al. 1998). The maser site in G 59.78+0.06 appears to coincide with a very weak radio source within $2^{\prime \prime}$ (Sridharan et al. 2002). In contrast, no radio continuum emission has been detected in G 173.49+2.42 and in G 188.95+0.89. Finally, the methanol maser in G 192.60-0.05 coincides with a 4.4-mJy radio source. Assuming that a maser site and a radio continuum source coincident in the maps are indeed co-spatial, the methanol masers in G 59.78+0.06 and G 192.60-0.05 are associated with the precursors of a UC H II region. In G 31.28+0.06, G 173.49+2.42 and G 188.95+0.89, further high angular resolution and high sensitivity $\mathrm{mm}$ continuum observations are required to identify the nature of the maser sources.

\subsection{Multiple star-forming (proto)clusters}

A secondary aspect of this work is the detection of objects only seen in mm continuum (hereafter "mm-only" clumps) and the variation of the SED profiles with the evolutionary status of the YSOs.

\subsubsection{Cold cores}

G59.78-MM1 and G 173.49-MM2 are typical mm-only clumps. G 31.28-MM3, G 173.49-MM3 and G 192.60-MM3 
are other possible mm-only clumps, making in total five mm-only clumps. G 188.95-MM2 probably emits in midIR, but its flux was not measured due to confusion with the main mid-IR source, G 188.95-MM1. These mm-only clumps are cold ( $20 \mathrm{~K}$ for G 173.49-MM2). The modelled luminosity in G 173.49-MM2 is only $300 L_{\odot}$. Assuming $T_{\text {cold }}=20 \mathrm{~K}$ for G 31.28-MM3, G 59.78-MM1, G 173.49MM3, G 192.60-MM3, masses of $\sim 25-178 M_{\odot}$ are derived for the five mm-only clumps. Excluding G 31.28-MM3 at $5.6 \mathrm{kpc}$, masses range 19-103 $M_{\odot}$ for G 59.78-MM1, G 173.49-MM2, G 173.49-MM3 and G 192.60-MM3. The modelled diameter of G $173.49-\mathrm{MM} 2$ is only $21 \times 10^{3} \mathrm{AU}$, i.e. $0.1 \mathrm{pc}$. Using an angular size of $24^{\prime \prime}$ for each object, column densities $\sim 2.5-7.4 \times$ $10^{22} \mathrm{~cm}^{-2}$ and visual extinction $\sim 26-78 \mathrm{mag}$ are estimated. The physical parameters of all detected mm-only clumps are summarised in Table 6.

The cold clumps might be prestellar clumps or protostellar clumps in the process of forming stars. Unfortunately, such types of clumps have marginally been detected and it is difficult to compare the mm-only clump properties with those of statistical samples of massive prestellar and early protostellar clumps. A good protostar candidate in an early accretion phase is IRAS 23385+6053 (Fontani et al. 2004), which is a cold $(\sim 40 \mathrm{~K})$ and small $(\sim 0.03 \mathrm{pc})$ molecular core embedded in a colder $(\sim 15 \mathrm{~K})$ and larger $(<0.4 \mathrm{pc})$ halo. The SED, luminosity $\left(150 L_{\odot}\right)$ and temperature $(15 \mathrm{~K})$ of IRAS $23385+6053$ resemble those of G173.49-MM2, when the IRAS points are excluded (Fig. 13 in Fontani et al. 2004). But as stated by the authors, it represents a lower limit. The luminosity of G 173.49-MM2 could also be underestimated without the knowledge of the far-IR flux.

\subsubsection{SED profiles}

In addition to the mm-only clumps, three other types of SED profile are identified. They might represent different evolutionary phases or result from geometrical effects that modify our perception of the YSO environments in function of the line-of-sight. G31.28-MM2 is the only example of a cold $(16 \mathrm{~K})$ envelope with a nearly hot $(115 \mathrm{~K})$ core. The envelope continuum emission and the core continuum emission peak at relatively low frequencies. G 59.78-MM2, G 173.49-MM4, G 188.95-MM1 and G 192.60-MM2 exhibit SEDs with a "warm" ( $45 \mathrm{~K})$ component peaking in the farIR at $60-100 \mu \mathrm{m}$ and a hot $(150-250 \mathrm{~K})$ component peaking in the mid-IR at $15-30 \mu \mathrm{m}$. They are all very luminous $\left(>10^{4} L_{\odot}\right)$. Finally, G 31.28-MM1, G173.49-MM1, G192.60MM1 and perhaps G 188.95-MM2 see their SED profile dominated by a strong far-IR component. The core component of the SED has shifted to longer wavelengths $(\sim 50 \mu \mathrm{m})$ and cooled down to $\sim 110 \mathrm{~K}$. The warm $(\sim 40 \mathrm{~K})$ envelope emission has strengthened and still peaks around $100 \mu \mathrm{m}$. The temperatures of all the "warm" components are in good agreement with those expected given the luminosities, sizes and $\beta$ (e.g. see Eq. (11) in Garay \& Lizano 1999). The hot component most likely probe the central part ( $d_{\text {hot }}=100$ to 3000 AU in Figs. $2-6$ ) of the core around the luminous protostellar object.
To compare the evolutionary status of each object, arguments are presented below and in Fig. 7. Only the clumps for which the SED can be built are considered. G 173.49-MM2 and G 31.28-MM2 present $L_{\text {submm }} / L_{\text {total }}>10^{-2}$ while the other clumps have $L_{\text {submm }} / L_{\text {total }}<3 \times 10^{-3}$, where $L_{\text {submm }}$ is the luminosity integrated between $1.2 \mathrm{~mm}$ and $450 \mu \mathrm{m}$. Similar results are noticed when considering $L_{\text {total }} / M_{\text {gas }}$ ratios, which are also independent of distances. G 173.49-MM2 and G31.28MM2 have lower $L_{\text {total }} / M_{\text {gas }}$ ratios $\left(3-10 L_{\odot} M_{\odot}^{-1}\right)$ than those measured for the other clumps $\left(>80 L_{\odot} M_{\odot}^{-1}\right)$. This could be explained if G 173.49-MM2 and G 31.28-MM2 are younger than the other objects associated with maser sites and UC H II regions, hence less luminous. This is also seen in Fig. 7top where G 173.49-MM2 (\#5 in Fig. 7) and G 31.28-MM2 (\#2 in Fig. 7) lie below the other sources whose luminosities follow $L_{\text {total }} \propto M_{\mathrm{gas}}^{0.75}$. The objects G 59.78-MM2, G 173.49MM4, G 188.95-MM1 and G 192.60-MM2 are all relatively hot inside, and strongly emit in the mid-IR with respect to the sub-mm emission (Fig. 7 bottom). They are all good HMC candidates. The mid-IR component is very much absorbed in the FIR component in the SEDs of G 31.28-MM1, G 173.49-MM1, G 188.95-MM2 and G 192.60-MM1. G 31.28MM1 and G 192.60-MM1 contain H II regions. The cases of G 173.49-MM1 and G 188.95-MM2 are unclear and they might belong to the HMC or UC H II class of objects.

Alternatively, the least massive clumps near the methanol maser site could be low- or intermediate-mass star-forming protoclusters. Their physical characteristic resemble those of the $\rho$-Oph mm cores (Motte et al. 1998). Indeed G 173.49-MM4 is coincident with S233IR NE, a cluster of lowmass YSOs seen in near-IR (Porras et al. 2000). The derived luminosity $\left(6 \times 10^{3} L_{\odot}\right)$ and mass $\left(20 M_{\odot}\right)$ are typical of the mm cores in $\rho$-Oph. In Fig. 7-middle, G 173.49-MM4 appears slightly isolated from the group of clearly identified massive star-forming clumps for which $F_{14}>F_{8}$ and $F_{21} / F_{8}>2$ (e.g. Lumsden et al. 2002). G $173.49+2.42$ is therefore a good example of a star-forming complex in which various mass types of YSO clusters co-exist.

High resolution and high sensitivity observations in mid-IR, $\mathrm{mm}$ and $\mathrm{cm}$ are needed to differentiate between these two scenarii by searching for multiple mm core system within each mm clump as seen in $\rho$-Oph and weak free-free emission around high-mass protostars.

\section{Conclusions}

Five methanol maser sites have been studied from mid-IR to $\mathrm{mm}$ wavelengths. Each radio-quiet maser site is always associated with a massive $\left(>50 M_{\odot}\right)$, very luminous $\left(>10^{4} L_{\odot}\right)$, deeply embedded ( $>40 \mathrm{mag})$ molecular clump. They are all underluminous stellar objects $\left(L_{\text {total }} \propto M_{\text {gas }}^{0.75}\right)$. The properties of the maser sites derived from observations and modelling suggest that they are embedded HMCs in which high-mass stars are in the process of forming. All these elements demonstrate that the radio-quiet methanol masers trace high-mass star-forming protoclusters in earlier phases than those detected in the H II region surveys. 

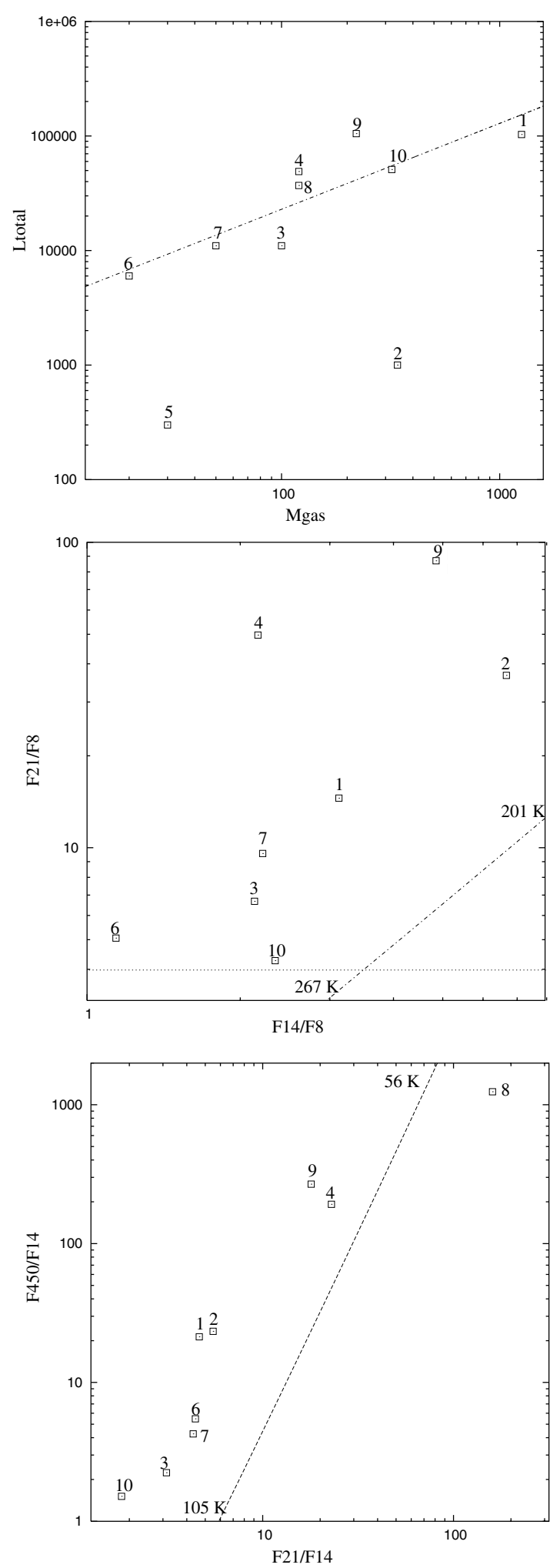

Fig. 7. Luminosity versus mass and colour diagrams. Each number represents an object as follows G 31.28-MM1(\#1), G 31.28-MM2(\#2), G 59.78-MM2(\#3), G 173.49-MM1(\#4), G 173.49-MM2(\#5), G 173.49-MM4(\#6), G188.95-MM1(\#7), G 188.95-MM2(\#8), G 192.60-MM1(\#9), G 192.60-MM2(\#10). The dashed line in the top plot represents $L_{\text {total }} \propto M_{\mathrm{gas}}^{0.75}$. The dashed lines in the colour diagrams represent a black body function with the temperature ranges indicated on the plots. The dotted line in the middle plot represents the massive YSO colour criterion from Lumsden et al. (2002).
In addition, colder gas clumps seen only at mm wavelengths are also found near the methanol maser sites. These might represent an even earlier phase of massive star formation. These results suggest an evolutionary sequence for massive star formation from a cold clump only seen at $\mathrm{mm}$ wavelengths, evolving to a HMC with a two-component SED with peaks at far-IR and mid-IR wavelengths, and ending with (ultra-compact) H II regions very bright at far-IR wavelengths. Alternatively, the cold clumps might be clusters of less massive $\left(<8 M_{\odot}\right)$ YSOs, in formation near the high-mass star-forming protoclusters.

Finally, the values of the dust grain emissivity index $(\beta)$ range between 1.6 and 1.9, which agree with modelling work (Ossenkopf \& Henning 1994).

Acknowledgements. This research was funded by an Australia Research Council Discovery Grant. It has also made use of Centre de Données astronomiques de Strasbourg (CDS), the Digitized Sky Survey produced at the Space Telescope Science Institute, the NASA/IPAC Infrared Science Archive operated by the Jet Propulsion Laboratory, California Institute of Technology. We thank the SEST staff for their help during the SIMBA observation and data reduction, especially Markus Nielbock for his data analysis support. The SEST and the Onsala-20 m telescope are operated by the Swedish National Facility for Radio Astronomy, Onsala Space Observatory at Chalmers University of Technology. The Mopra millimetre radio-telescope is part of the Australia Telescope which is funded by the Commonwealth of Australia for operation as a National Facility managed by CSIRO.

\section{References}

Batrla, W., Matthews, H., Menten, K., \& Walmsley, C. 1987, Nature, 326, 49

Becker, R. H., White, R. L., Helfand, D. J., \& Zoonematkermani, S. 1994, ApJS, 91, 347

Beuther, H., Schilke, P., Menten, K. M., et al. 2002, ApJ, 566, 945

Bourke, T. L., Garay, G., Lehtinen, K. K., et al. 1997, ApJ, 476, 781

Carpenter, J. M., Meyer, M. R., \& Dougados, C. 1997, AJ, 114, 198

Caswell, J. L., Vaile, R. A., Ellingsen, S. P., Whiteoak, J. B., \& Norris, R. P. 1995, MNRAS, 272, 96

Caswell, J. L. 1996, MNRAS, 279, 79

Condon, J. J., Cotton, W. D., Greisen, E. W., et al. 1998, AJ, 115, 1693

Egan, M. P., Price, S. D., Moshir, M. M., Cohen, M., \& Tedesco, E. 1999, The Midcourse Space Experiment Point Source Catalog version 1.2 Explanatory Guide, Technical Report, AD-A381933

Fontani, F., Cesaroni, R., Testi, L., et al. 2004, A\&A, 414, 299

Frerking, M. A., Langer, W. D., \& Wilson, R. W. 1982, ApJ, 262, 590

Garay, G., \& Lizano, S. 1999, PASP, 111, 1049

Greene, T. P., \& Young, E. T. 1989, ApJ, 339, 258

Gregory, P. C., Scott, W. K., Douglas, K., \& Condon, J. J. 1996, ApJS, 103,427

Hildebrand, R. H. 1983, QJRAS, 24, 267

Hofner, P., Cesaroni, R., Rodríguez, L. F., \& Martí, J. 1999, A\&A, 345, L43

Israel, F. P., \& Felli, M. 1978, A\&A, 63, 325

Kalenskii, S. V., Promislov, V. G., Alakoz, A., Winnberg, A. V., \& Johansson, L. E. B. 2000, A\&A, 354, 1036

Kurtz, S., Churchwell, E., \& Wood, D. O. S. 1994, ApJS, 91, 659

Kurtz, S., Cesaroni, R., Churchwell, E., Hofner, P., \& Walmsley, M. 2000, in Protostars and Planets IV, ed. V. Mannings, A. P. Boss, \& S. S. Russell (University of Arizona Press), 299 
Lada, C. J., \& Lada, E. A. 2003, ARA\&A, 41, 57

Lockman, F. J. ApJS, 71, 469

Lumsden, S. L., Hoare, M. G., Oudmaijer, R. D., \& Richards, D. 2002, MNRAS, 336, 621

MacLaren, I., Richardson, K. M., \& Wolfendale, A. W. 1988, ApJ, 333,821

Megeath, S. T., Herter, T., Beichman, C., et al. 1996, A\&A, 307, 775

Minier, V., \& Booth, R. S. 2002, A\&A, 387, 179

Minier, V., Booth, R. S., \& Conway, J. E. 2000, A\&A, 362, 1093

Minier, V., Conway, J. E., \& Booth, R. S. 2001, A\&A, 369, 278

Minier, V., Ellingsen, S. P., Norris, R. P., \& Booth, R. S. 2003, A\&A, 403, 1095

Motte, F., André, P., \& Neri, R. 1998, A\&A, 336, 150

Nummelin, A. 1998, Ph.D. Thesis, Technical Report No. 347, Chalmers University of Technology

Olmi, L., Cesaroni, R., Hofner, P., et al. 2003, A\&A, 407, 225

Ossenkopf, V., \& Henning, T. 1994, A\&A, 291, 943

Panagia, N. 1973, AJ, 78, 929

Panagia, N., \& Felli, M. 1975, A\&A, 39, 1

Pestalozzi, M. R., Minier, V., \& Booth, R. S. 2004, A\&A, submitted
Phillips, C. J., Norris, R. P., Ellingsen, S. P., \& McCulloch, P. M. 1998, MNRAS, 300, 1131

Porras, A., Cruz-Gonzlez, I., \& Salas, L. 2000, A\&A, 361, 660

Purcell, C. R., Burton, M. G., Hunt, M., Minier, V., \& Hill, T. 2004, in Star Formation at High Angular Resolution, IAU Symp., 221, in press

Rodgers, S. D., \& Charnley, S. B. 2001, ApJ, 546, 324

Salter, C. J. 1983, The NRAO 12-m Telescope User's Guide

Schutte, A., van der Walt, D., Gaylard, M., \& MacLeod, G. 1993, MNRAS, 261, 783

Snell, R. L., \& Bally, J. 1986, ApJ, 303, 683

Sridharan, T. K., Beuther, H., Schilke, P., Menten, K. M., \& Wyrowski, F. 2002, 566, 931

van der Walt, D. J., Churchwell, E., Gaylard, M. J., \& Goedhart, S. 2003, MNRAS, 341, 270

Walsh, A., Burton, M., Hylard, A., \& Robinson, G. 1998, MNRAS, 301,640

Walsh, A. J., Macdonald, G. H., Alvey, N. D. S., Burton, M. G., \& Lee, J.-K. 2003, A\&A, 410, 597

Wilking, B. A., \& Lada, C. J. 1983, ApJ, 274, 571 